\title{
Almost all Steiner triple systems are almost resolvable
}

\author{
Asaf Ferber ${ }^{1}$ and Matthew Kwan ${ }^{2}$ \\ ${ }^{1}$ Department of Mathematics, University of California, Irvine, California, USA; E-mail: asaff@uci.edu. \\ ${ }^{2}$ Department of Mathematics, Stanford University, Stanford, California 94305, USA; E-mail: mattkwan@ stanford.edu.
}

Received: 21 July 2019; Revised: 30 July 2020; Accepted: 3 July 2020

2020 Mathematics Subject Classification: Primary - 35P15, Secondary - 58C40, 53A07

Keywords and phrases: survey design, measurement error, machine learning

\begin{abstract}
We show that for any $n$ divisible by 3, almost all order- $n$ Steiner triple systems admit a decomposition of almost all their triples into disjoint perfect matchings (that is, almost all Steiner triple systems are almost resolvable).
\end{abstract}

\section{Contents}

1 Introduction $\mathbf{2}$

1.1 Structure of the paper ........................... . . . . . . . . . . .

1.2 Notation ................................. 4

2 Random Steiner triple systems via the triangle removal process 4

2.1 A coupling lemma . . . . . . . . . . . . . . . . . . . . . . . 5

3 Sufficient properties for packing 6

4 Sparse regularity and the KER conjecture for hypergraphs 9

4.1 Refining an existing partition ........................10

4.2 The KER conjecture . . . . . . . . . . . . . . . . . . . . . . . 12

5 Almost-perfect matchings 12

6 Packing in good systems 13

6.1 Partitioning for packing . . . . . . . . . . . . . . . . . . . . 13

6.2 Absorbers ................................. 15

6.3 Proof of Lemma 3.6 . . . . . . . . . . . . . . . . . . . . . . . . 16

7 Goodness in random Steiner triple systems 17

7.1 Almostregularity ........................... 17

7.2 Upper-quasirandomness . . . . . . . . . . . . . . . . . . . . . 17

7.3 Embedding absorbers ......................... 17

8 Concluding remarks $\quad \mathbf{2 1}$

Appendix A Adapting the proof of the KER conjecture to linear hypergraphs 22

(C) The Author(s), 2020. Published by Cambridge University Press. This is an Open Access article, distributed under the terms of the Creative Commons Attribution licence (http://creativecommons. org/licenses/by/4.0/), which permits unrestricted re-use, distribution, and reproduction in any medium, provided the original work is properly cited. 


\section{Introduction}

One of the oldest problems in combinatorics, posed by Kirkman in 1850 [24] is the following:

Fifteen young ladies in a school walk out three abreast seven days in succession: it is required to arrange them daily so that no two shall walk twice abreast.

This problem was solved by Kirkman a few years earlier [23] (although the first published solution is due to Cayley [6]).

In order to frame the above question in more generality, we need to introduce some terminology. An order $n$ Steiner triple system (STS $(n)$ for short) is a collection of triples of $[n]:=\{1, \ldots, n\}$ for which every pair of elements is contained in exactly one triple. These objects are named after Jakob Steiner, who observed the existence of such systems in 1853 (it is an interesting historical note that this happened after Kirkman proposed his problem).

As an example, observe that the collection $\{123,145,167,246,257,347,356\}$ forms an STS(7), and a simple counting argument yields that an $\mathbf{S T S}(n)$ can only exist if $n \equiv(1$ or 3$)$ mod 6. It is also known that for every such $n$, a construction of an $\operatorname{STS}(n)$ can be achieved. The study of Steiner triple systems (and some natural generalisations, such as Steiner systems and block designs) has a long and rich history. These structures have strong connections to a wide range of different subjects, ranging from group theory, to finite geometry, to experimental design, to the theory of error-correcting codes, and more. For an introduction to the subject, the reader is referred to [7].

Now, for an order- $n$ Steiner triple system $S$, a matching in $S$ is a collection of disjoint triples of $S$, and a perfect matching (also known as a resolution class or parallel class) is a matching covering the entire ground set $[n]$. Observe that a perfect matching consists of exactly $\frac{n}{3}$ triples and therefore can exist only when $n=3 \bmod 6$. We say that $S$ is resolvable (or has parallelism) if the triples in $S$ can be perfectly partitioned into perfect matchings. Since $S$ has $\left(\begin{array}{l}n \\ 2\end{array}\right) / 3$ triples and every perfect matching has $n / 3$ edges, such a partition must consist of exactly $\frac{n-1}{2}$ perfect matchings.

Using the above terminology, Kirkman's problem is simply asking whether there exists a resolvable STS(15). More generally, one can ask whether there exists a resolvable $\operatorname{STS}(n)$ for any $n=3 \bmod 6$. This problem was famously solved in the affirmative by Ray-Chaudhuri and Wilson [39] in 1971, over 100 years after Kirkman posed his problem.

Despite the difficulty in proving even that resolvable Steiner triple systems exist, the existence of many large matchings seems to actually be a 'typical' property of Steiner triple systems. Early results were based on Rödl-nibble ${ }^{1}$ type arguments. A result of Pippenger and Spencer [37] shows that every $\operatorname{STS}(n)$ contains disjoint matchings of size $n-o(n)$ that cover a $(1-o(1))$-fraction of its triples; and Alon, Kim, and Spencer [1] later proved that every $\mathbf{S T S}(n)$ contains at least one matching that covers all but at most $O\left(n^{1 / 2} \log ^{3 / 2} n\right)$ elements. More recently, building on the breakthrough work of Keevash [19] concerning the existence and completion of block designs, Kwan [31] proved that if $n=3$ mod 6, then almost all (meaning a $(1-o(1))$-fraction of) order- $n$ Steiner triple systems have a perfect matching. In fact, almost every $\mathbf{S T S}(n)$ has $\left((1-o(1)) n /\left(2 e^{2}\right)\right)^{n / 3}$ different perfect matchings. This proof was adapted by Morris [36] to show that almost every $\operatorname{STS}(n)$ has $\Omega(n)$ disjoint perfect matchings. We would go so far as to make the following conjecture:

Conjecture 1.1. For $n=3 \bmod 6$, almost every $\operatorname{STS}(n)$ is resolvable.

We remark that in Conjecture 1.1, 'almost every' certainly cannot be replaced with 'every': Bryant and Horsley [4] proved that for infinitely many $n=3 \bmod 6$, there exist Steiner triple systems with not even a single perfect matching.

Of course, Conjecture 1.1 and the aforementioned result of Kwan are really about random Steiner triple systems: we are trying to understand properties that hold a.a.s. ${ }^{2}$ in a uniformly random $\operatorname{STS}(n)$.

\footnotetext{
1The 'nibble' is a type of probabilistic argument introduced by Rödl [40] for finding almost-perfect matchings in set systems. Far beyond the study of Steiner triple systems, it has had significant influence throughout combinatorics in the last 30 years.

2By 'asymptotically almost surely' (a.a.s.), we mean that the probability of an event is $1-o(1)$. Here and for the rest of the paper, asymptotics are as $n \rightarrow \infty$.
} 
This turns out to be surprisingly difficult: despite the enormous advances in the theory of random combinatorial structures since the foundational work of Erdôs and Rényi [10], almost none of the available tools seem to be applicable to random Steiner triple systems. Random Steiner triple systems lack independence or any kind of recursive structure, which rules out many of the techniques used to study Erdôs-Rényi random graphs and random permutations; and there is basically no freedom to make local changes, which precludes the use of 'switching' techniques often used in the study of random regular graphs (see, for example, [29]). It is not even clear how to study a random STS $(n)$ empirically: in an attempt to find an efficient algorithm to generate a random STS $(n)$, Cameron [5] designed a Markov chain on Steiner triple systems, but he was not able to determine whether this chain was connected. As far as we know, before the work of Kwan [31], the only nontrivial fact known to hold for a random $\operatorname{STS}(n)$ was that it a.a.s. has a trivial automorphism group, a fact proved by Babai [2] using a direct (and rather coarse) counting argument.

Building on the ideas in [31], introducing the sparse regularity method, and extending a random partitioning argument from [11], in this paper we prove the following 'asymptotic' version of Conjecture 1.1 , adding to the short list of known facts about random Steiner triple systems.

Theorem 1.2. Let $\boldsymbol{S}$ be a uniformly random $\boldsymbol{S T S}(n)$, where $n=3 \bmod 6$. Then a.a.s. $\boldsymbol{S}$ has $(1 / 2-o(1)) n$ disjoint perfect matchings.

Our proof of Theorem 1.2 is based on tools and intuition from random hypergraph theory. We conclude this introduction with a brief discussion of random hypergraphs and some related work.

For $k \geq 2$, a $k$-uniform hypergraph $H$ (or a $k$-graph for short) is a pair $H=(V, E)$, where $V$ is a finite set of vertices and $E \subseteq\left(\begin{array}{c}V \\ k\end{array}\right)$ is a family of $k$-element subsets of $V$, referred to as edges. The existence of perfect matchings is one of the most central questions in the theory of graphs and hypergraphs. In the case of graphs (that is, 2-uniform hypergraphs), the problem of finding a perfect matching (if one exists) is relatively simple, but the analogous problem in the hypergraph setting is known to be NP-hard (see [18]). Therefore, a main theme in extremal and probabilistic combinatorics is to investigate sufficient conditions for the existence of perfect matchings in hypergraphs. The results that are most relevant to our paper are those regarding the problem of finding perfect matchings in random hypergraphs.

Let $\mathrm{H}^{k}(n, p)$ be the (binomial) random $k$-uniform hypergraph distribution on the vertex set $V(H)=$ $[n]$, where each $k$-set $X \in\left(\begin{array}{c}{[n]} \\ k\end{array}\right)$ is included as an edge with probability $p$ independently. A main problem in this area was to find a 'threshold' function $q(n)$ for the property of containing a perfect matching: that is, to find a function $q$ for which, when $\boldsymbol{G} \sim \mathrm{H}^{k}(n, p)$, we have

$$
\operatorname{Pr}[\boldsymbol{G} \text { contains a perfect matching }] \rightarrow \begin{cases}1 & \text { if } p(n) / q(n) \rightarrow \infty \\ 0 & \text { if } p(n) / q(n) \rightarrow 0\end{cases}
$$

Following a long line of work, the most significant development in this area is the celebrated work of Johansson, Kahn, and $\mathrm{Vu}$ [17] where they showed that $q(n)=\log n / n$ defines a threshold function for this property. Two other relevant results are those of Frieze and Krivelevich [13] and its variant due to Ferber, Kronenberg, and Long [11], which establish a way to find 'many' edge-disjoint perfect matchings/Hamiltonian cycles in random (hyper)graphs based on some pseudorandom properties and a random partitioning argument.

\subsection{Structure of the paper}

The structure of the paper is as follows. In Section 2, we review the methods introduced in [31] for studying random Steiner triple systems via the triangle removal process and present one new lemma for studying monotone decreasing properties. In Section 3, we outline the general approach of the proof, stating some properties that we will use to pack perfect matchings and that we will be able to show hold a.a.s. in a random Steiner triple system. 
Before turning to the proof of Theorem 1.2, in Section 4 we discuss the sparse regularity lemma for hypergraphs and some auxiliary lemmas for applying it; and in Section 5, we discuss some lemmas for finding almost-perfect matchings in hypergraphs.

The proof of Theorem 1.2 itself appears in Sections 6 and 7. In Section 6 we prove that certain properties suffice for packing perfect matchings, and in Section 7 we show that these properties a.a.s. hold in random Steiner triple systems.

Finally, in Section 8 we have some concluding remarks; and in Section Appendix A, we explain how to generalise the proof by Conlon, Gowers, Samotij, and Schacht [8] of the so-called KŁR conjecture to 'linear' hypergraphs. This will be a key tool in our proof.

\subsection{Notation}

We use standard asymptotic notation throughout, as follows. For functions $f=f(n)$ and $g=g(n)$, we write $f=O(g)$ to mean that there is a constant $C$ such that $|f| \leq C|g|, f=\Omega(g)$ to mean that there is a constant $c>0$ such that $f(n) \geq c|g(n)|$ for sufficiently large $n, f=\Theta(g)$ to mean that that $f=O(g)$ and $f=\Omega(g)$, and $f=o(g)$ to mean that $f / g \rightarrow 0$ as $n \rightarrow \infty$. Also, following [20], the notation $f=1 \pm \varepsilon$ means $1-\varepsilon \leq f \leq 1+\varepsilon$.

We also use standard graph theory notation: $V(G)$ and $E(G)$ are the sets of vertices and (hyper)edges of a (hyper)graph $G$, and $v(G)$ and $e(G)$ are the cardinalities of these sets. The subgraph of $G$ induced by a vertex subset $U$ is denoted $G[U]$, the degree of a vertex $v$ is denoted $\operatorname{deg}_{G}(v)$, and the subgraph obtained by deleting $v$ is denoted $G-v$. Given a subset of vertices $U \subseteq V(G)$ and any vertex $v \in V(G)$, we let $\operatorname{deg}_{U}(v)$ denote the degree of $v$ into the subset $U$ (that is, the number of edges consisting of $v$ and some vertices of $U$ ).

For a positive integer $n$, we write $[n]$ for the set $\{1,2, \ldots, n\}$. For a real number $x$, the floor and ceiling functions are denoted $\lfloor x\rfloor=\max \{i \in \mathbb{Z}: i \leq x\}$ and $\lceil x\rceil=\min \{i \in \mathbb{Z}: i \geq x\}$. We will, however, mostly omit floor and ceiling signs and assume that large numbers are integers wherever divisibility considerations are not important. We will use the convention that random objects (for example, random variables or random graphs) are printed in bold. Finally, all logarithms are in base $e$.

\section{Random Steiner triple systems via the triangle removal process}

In this section, we reproduce the general theorems from [31] for studying the behaviour of a randomly chosen $\mathbf{S T S}(n)$ via the triangle removal process, including a new lemma for studying monotone decreasing properties. This machinery will be crucial to prove Theorem 1.2.

Note that any $\operatorname{STS}(n)$ is a 3-graph, and let $N=\left(\begin{array}{c}n \\ 2\end{array}\right) / 3=(1+o(1)) n^{2} / 6$ be the number of edges in any $\operatorname{STS}(n)$. We assume throughout this section that $n$ is 1 or $3 \bmod 6$ (as otherwise, an $\mathbf{S T S}(n)$ does not exist). Let us first make some useful definitions.

Definition 2.1 (partial Steiner triple systems). A partial Steiner triple system (also known as a linear 3-graph) is a 3-graph on the vertex set $[n]$ in which every pair of vertices is included in at most one edge. We will also want to consider partial Steiner triple systems equipped with an ordering on their edges. Let $\mathcal{O}$ be the set of ordered Steiner triple systems, and let $\mathcal{O}_{m}$ be the set of ordered partial Steiner triple systems with exactly $m$ edges. For $S \in \mathcal{O}_{m}$ and $i \leq m$, let $S_{i}$ be the ordered partial Steiner triple system consisting of just the first i edges of $S$. For a (possibly ordered) partial Steiner triple system $S$, let $G(S)$ be the graph (that is, a 2-graph) with an edge for every pair of vertices that does not appear in any edge of $S$. So, if $S$ has $m$ edges, then $G(S)$ has $\left(\begin{array}{l}n \\ 2\end{array}\right)-3 m$ edges.

Definition 2.2 (quasirandomness). For a graph $G$ with $n$ vertices and $m$ edges, let $d(G)=m /\left(\begin{array}{l}n \\ 2\end{array}\right)$ denote its density. We say $G$ is $(\varepsilon, h)$-quasirandom if for every set $A$ of at most $h$ vertices, we have $\left|\bigcap_{w \in A} N_{G}(w)\right|=(1 \pm \varepsilon) d(G)^{|A|} n$. Let $\mathcal{O}_{m}^{\varepsilon, h} \subseteq \mathcal{O}_{m}$ be the set of ordered partial Steiner triple systems $S \in \mathcal{O}_{m}$ such that $G\left(S_{i}\right)$ is $(\varepsilon, h)$-quasirandom for each $i \leq m$. 
Definition 2.3 (the triangle removal process). The triangle removal process is defined as follows. Start with the complete graph $K_{n}$, and iteratively delete the edge-set of a triangle chosen uniformly at random from all triangles in the remaining graph. If we continue this process for $m$ steps, the deleted triangles (in order) can be interpreted as an ordered partial Steiner triple system in $\mathcal{O}_{m}$. It is also possible that the process aborts (because there are no triangles left) before $m$ steps, in which case we say it returns the value ' $*$ '. We denote by $\mathrm{R}(n, m)$ the resulting distribution on $\mathcal{O}_{m} \cup\{*\}$.

Now we can state the general theorem from [31] comparing a typical $\operatorname{STS}(n)$ with a typical outcome of the triangle removal process. Basically, if we can show that the first few edges of the triangle removal process (as an ordered partial Steiner triple system) satisfy some property with extremely high probability, then it follows that the first few edges of a uniformly random ordered STS $(n)$ satisfy the same property with high probability. Moreover, it suffices to study the triangle removal process conditioned on some 'good' event, provided that this event contains the event that (the graph of uncovered edges of) our partial Steiner triple system is sufficiently quasirandom.

Theorem 2.4. Fixing $h \in \mathbb{N}$ and sufficiently small $a>0$, there is $b=b(a, h)>0$ such that the following holds. Fix $\alpha \in(0,1)$, let $\mathcal{P} \subseteq \mathcal{O}_{\alpha N}$ be a property of ordered partial Steiner triple systems, let $\varepsilon=n^{-a}$, let $\mathcal{Q} \supseteq \mathcal{O}_{\alpha N}^{\varepsilon, h}$, let $\boldsymbol{S} \in \mathcal{O}$ be a uniformly random ordered Steiner triple system and let $\boldsymbol{S}^{\prime} \sim \mathrm{R}(n, \alpha N)$. If

$$
\operatorname{Pr}\left(\boldsymbol{S}^{\prime} \notin \mathcal{P} \mid \boldsymbol{S}^{\prime} \in \mathcal{Q}\right) \leq \exp \left(-n^{2-b}\right)
$$

then

$$
\operatorname{Pr}\left(\boldsymbol{S}_{\alpha N} \notin \mathcal{P}\right) \leq \exp \left(-\Omega\left(n^{1-2 a}\right)\right)
$$

\subsection{A coupling lemma}

The triangle removal process can be rather technical to study directly, so [31] included a general lemma approximating the first few steps of the process with a binomial random 3-graph with relatively small edge probability. The idea is that instead of randomly choosing triples one by one, avoiding conflicts with previous choices, one can randomly choose several triples at once and just delete those triples that conflict with each other. If the edge probability is small, there are likely to be few conflicts, so these two processes (at least intuitively) give almost the same distribution.

This lemma (specifically, [31, Lemma 2.10]) was suitable for studying properties $\mathcal{P}$ that are monotone increasing in the sense that $S \in \mathcal{P}$ and $S^{\prime} \supseteq S$ implies $S^{\prime} \in \mathcal{P}$. Surprisingly, despite the fact that the existence of perfect matchings (or collections of disjoint perfect matchings) is a monotone increasing property, in this paper we will instead need a similar lemma for monotone decreasing properties. Before stating the lemma, we need the following definition.

Definition 2.5. For a partial Steiner triple system $S$, let $\mathrm{R}(S, m)$ be the partial Steiner triple system distribution obtained with $m$ steps of the triangle removal process starting from $G(S)$. (So, if $S$ has $m^{\prime}$ edges, then $\mathrm{R}(S, m)$ has $m+m^{\prime}$ edges, unless the triangle removal process aborts.)

Lemma 2.6. Fix sufficiently small $\alpha \in(0,1)$. Consider some $S \in \mathcal{O}_{m}^{\alpha, 2}$ for some $m \leq \alpha N$, and consider a property $\mathcal{P}$ of unordered 3-graphs (which may depend on $S$ ) that is monotone decreasing in the sense that $G \in \mathcal{P}$ and $G^{\prime} \subseteq G$ implies $G^{\prime} \in \mathcal{P}$. Let $\boldsymbol{S} \sim \mathrm{R}(S, \alpha N)$ and $\boldsymbol{G} \sim \mathrm{H}^{3}(n, q)$ with $q=\alpha(1+10 \alpha) / n$. Then

$$
\operatorname{Pr}(\boldsymbol{S} \notin \mathcal{P}) \leq \operatorname{Pr}(\boldsymbol{G} \notin \mathcal{P})+e^{-\Omega\left(n^{2}\right)}
$$

The proof of Lemma 2.6 is somewhat more complicated than [31, Lemma 2.10], but the intuition is basically the same: if $\alpha$ is small and $S$ is a partial Steiner triple system with few edges, then the outcome of $\mathrm{H}^{3}(n, q)$ is likely to 'almost' be a partial Steiner triple system and 'almost' avoid conflicts with $S$, and therefore approximates $\mathrm{R}(S, \alpha N)$. For the proof we will need the following concentration inequality, 
which appears as [31, Theorem 2.11], and is also a direct consequence of [45, Theorem 1.3]. It is a bounded-differences inequality with Bernstein-type tails that can be used to analyse sparse random hypergraphs. Standard bounded-difference inequalities such as the Azuma-Hoeffding inequality do not provide strong enough tail bounds to apply Lemma 2.4 .

Theorem 2.7. Let $\omega=\left(\omega_{1}, \ldots, \omega_{n}\right)$ be a sequence of independent, identically distributed random variables with $\operatorname{Pr}\left(\omega_{i}=1\right)=p$ and $\operatorname{Pr}\left(\omega_{i}=0\right)=1-p$. Let $f:\{0,1\}^{n} \rightarrow \mathrm{R}$ satisfy the Lipschitz condition $\left|f(\omega)-f\left(\omega^{\prime}\right)\right| \leq K$ for all pairs $\omega, \omega^{\prime} \in\{0,1\}^{n}$ differing in exactly one coordinate. Then

$$
\operatorname{Pr}(|f(\omega)-\mathbb{E} f(\omega)|>t) \leq \exp \left(-\frac{t^{2}}{4 K^{2} n p+2 K t}\right) .
$$

We will also need the fact that quasirandom graphs have approximately the 'right' number of triangles.

Lemma 2.8. Let $\varepsilon$ be sufficiently small, and let $G$ be an $(\varepsilon, 2)$-quasirandom graph with density $d$. Then $G$ has $(1 \pm 3 \varepsilon) d^{3} n^{3} / 6$ triangles.

Proof. By $(\varepsilon, 1)$-quasirandomness, the sum of the degrees is $(1 \pm \varepsilon) d n^{2}$, so there are $(1 \pm \varepsilon) d n^{2} / 2$ edges. For each such edge, by $(\varepsilon, 2)$-quasirandomness, there are $(1 \pm \varepsilon) d^{2} n$ common neighbours of that edge, each of which gives a triangle containing that edge. Therefore, the total number of triangles is

$$
\left((1 \pm \varepsilon) d n^{2} / 2\right)\left((1 \pm \varepsilon) d^{2} n\right) / 3=(1 \pm 3 \varepsilon) d^{3} n^{3} / 6
$$

as desired.

Now we can prove Lemma 2.6.

Proof of Lemma 2.6. Let $\boldsymbol{S}^{*}$ be obtained from $\boldsymbol{G}$ by deleting every edge that intersects an edge of $S$ or another edge of $\boldsymbol{G}$ in two vertices. We can couple $\left(\boldsymbol{S}^{*}, \boldsymbol{G}\right)$ and $\boldsymbol{S}$ in such a way that $\boldsymbol{G} \supseteq \boldsymbol{S}$ as long as $\boldsymbol{S}^{*}$ has at least $\alpha N$ edges (randomly order the edges in $\boldsymbol{G}$, and run the triangle removal process with this ordering). Let $\boldsymbol{Y}$ be the number of edges in $\boldsymbol{S}^{*}$, which is the number of edges in $\boldsymbol{G}$ that do not conflict with $S$ and are isolated in the sense that they do not intersect any other edge of $\boldsymbol{G}$ in more than one vertex. By Lemma 2.8, there are at least $(1 \pm 3 \alpha)(1-\alpha)^{3} n^{3} / 6=(1 \pm 7 \alpha) n^{3} / 6$ possibilities for such an edge, and each is present and isolated with probability

$(\alpha(1+10 \alpha) / n)(1-\alpha(1+10 \alpha) / n)^{3(n-3)+1}=\alpha(1+10 \alpha-o(1)) e^{-3 \alpha(1+10 \alpha)} / n=\left(1+7 \alpha+O\left(\alpha^{2}\right)\right) \alpha / n$

for sufficiently small $\alpha$. This implies that $\mathbb{E} \boldsymbol{Y}=\alpha N+\Omega\left(n^{2}\right)$. Next, observe that adding an edge to $\boldsymbol{G}$ can increase $\boldsymbol{Y}$ by at most 1, and removing an edge to $\boldsymbol{G}$ can increase $\boldsymbol{Y}$ by at most 3 (by making three edges isolated). So, by Theorem 2.7 ,

$$
\operatorname{Pr}(\boldsymbol{Y}<\alpha N) \leq \operatorname{Pr}\left(|\boldsymbol{Y}-\mathbb{E} Y| \geq \Omega\left(n^{2}\right)\right) \leq \exp \left(-\Omega\left(\frac{\left(n^{2}\right)^{2}}{3^{2}\left(\begin{array}{c}
n \\
3
\end{array}\right)(\alpha(1+10 \alpha) / n)+3 n^{2}}\right)\right)=e^{-\Omega\left(n^{2}\right)} .
$$

It follows that

$$
\operatorname{Pr}(\boldsymbol{S} \notin \mathcal{P}) \leq \operatorname{Pr}(\boldsymbol{G} \notin \mathcal{P})+\operatorname{Pr}(\boldsymbol{Y}<\alpha N)=\operatorname{Pr}(\boldsymbol{G} \notin \mathcal{P})+e^{-\Omega\left(n^{2}\right)}
$$

\section{Sufficient properties for packing}

In this section, we state some lemmas from which Theorem 1.2 will follow. Basically, the idea is to define some properties that can be shown to hold a.a.s. in random Steiner triple systems and that can be used to find 'many' perfect matchings. The first of these properties is an 'upper-quasirandomness' condition, which is a requirement to effectively apply the so-called regularity method in the sparse setting. 


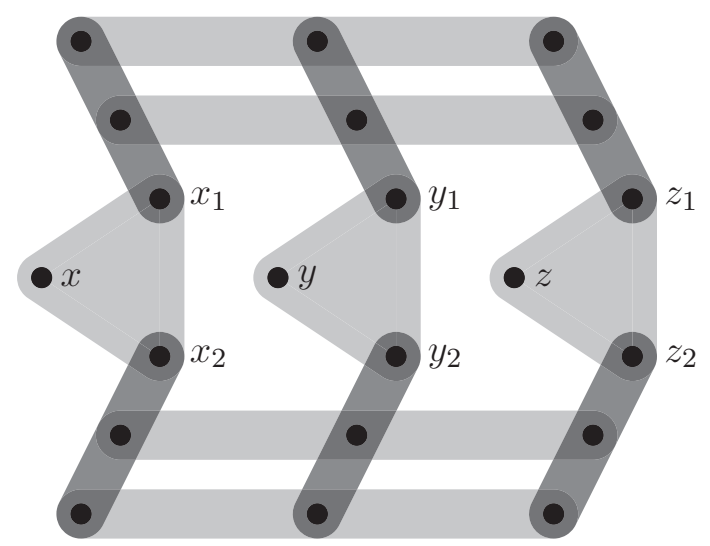

Figure 1. An illustration of an absorber for $(x, y, z)$. The light edges are the covering matching, and the dark edges are the non-covering matching.

Definition 3.1. For vertex sets $X, Y, Z$ in a 3-graph $G$, let $e_{G}(X, Y, Z)$ be the number of orderings $(x, y, z)$ of edges $\{x, y, z\}$ with $x \in X, y \in Y, z \in Z$ (if $X, Y, Z$ are disjoint, this is the number of edges with exactly one vertex in each of $X, Y, Z)$. A 3-graph is $(p, \beta)$-upper-quasirandom if for any vertex subsets $X, Y, Z$, we have $e(X, Y, Z) \leq p|X||Y||Z|+\beta n^{3} p$.

The second property we will need is the existence of certain special subgraphs that we refer to as absorbers. We will later see that it is not very hard to construct 'almost'-perfect matchings, and we will be able to use the special features of absorbers to complete almost-perfect matchings into perfect matchings (by 'absorbing' the uncovered vertices). This idea falls into the framework of the absorption method, which was first introduced as a general method by Rödl, Ruciński and Szemerédi in [41] and has had numerous applications since.

Definition 3.2 (sub-absorbers and absorbers). The absorbers we use (see also Figure 1 for an illustration) are based on sub-absorbers and will be defined in two steps:

- A sub-absorber rooted on a triple of vertices $x, y, z$ is a set of five edges of the form

$$
\left\{\left\{x, x_{1}, x_{2}\right\},\left\{y, y_{1}, y_{2}\right\},\left\{z, z_{1}, z_{2}\right\},\left\{x_{1}, y_{1}, z_{1}\right\},\left\{x_{2}, y_{2}, z_{2}\right\}\right\}
$$

We call $x, y, z$ the rooted vertices of the sub-absorber, and we call the other nine vertices the external vertices. If an edge contains a rooted vertex, we call it a rooted edge.

- An absorber rooted on a triple of vertices $x, y, z$ is a set of 13 edges obtained in the following way.

Put three disjoint edges $\left\{x, x_{1}, x_{2}\right\},\left\{y, y_{1}, y_{2}\right\},\left\{z, z_{1}, z_{2}\right\}$, then put a sub-absorber rooted on $\left\{x_{1}, y_{1}, z_{1}\right\}$ and a sub-absorber rooted on $\left\{x_{2}, y_{2}, z_{2}\right\}$ (in such a way that no pair of edges intersects in more than one vertex). We call $x, y, z$ the rooted vertices of the absorber and the other 18 vertices the external vertices. If an edge contains a rooted vertex, we call it a rooted edge. Note that the edges of an absorber can be partitioned into two matchings: a perfect matching with seven edges (which we call the covering matching) and a matching with six edges (which we call the non-covering matching).

The crucial property of an absorber that allows it to be used to complete a matching into a perfect matching is the existence of the covering and non-covering matching (we can choose whether to cover the root vertices $x, y, z$ ). The observant reader may notice that sub-absorbers are simpler structures also having a covering and non-covering matching; the reason we consider the larger absorbers is that it is easier to find a rooted absorber in a random 3-graph than a rooted sub-absorber (primarily because the rooted edges in an absorber have no common incident edges). 
In the proof of our main result, we will want to construct our perfect matchings one by one, so it will be important that we can find absorbers disjoint to a set of perfect matchings that have already been constructed. To do so, we will show that the 3-graphs we are working with are 'resilient' with respect to absorbers, in the sense that every 'dense' subgraph contains at least one absorber for each triple.

Definition 3.3. A 3-graph $G$ is $D$-absorber-resilient if every subgraph $G^{\prime} \subseteq G$ with minimum degree at least $D$ has an absorber rooted on every triple $x, y, z$ of distinct vertices of $G$.

Now, the properties we will use for packing perfect matchings are as follows. It will be useful for the reader to think of $\beta$ as a very small constant that controls the error terms and of $\alpha$ as a constant that is much larger (but still quite small in absolute terms) that measures the fraction of a Steiner triple system we are considering.

Definition 3.4. Consider $\alpha, \beta>0$, and let $S$ be any 3-graph. We say that $S$ is $(\alpha, \beta)$-good if it satisfies the following properties:

1. Almost-regularity: For every vertex $v$, we have $\operatorname{deg}_{S}(v)=\alpha n / 2 \pm \beta n$.

2. Pseudorandomness: $S$ is $(\alpha / n, \beta)$-upper-quasirandom.

3. Robust absorber-resilience: For each $w \geq \beta$, letting $\eta=w / n$, all but at most

$$
\exp \left(-10^{-8}(w / n)^{4} \alpha^{2} n\right)\left(\begin{array}{l}
n \\
w
\end{array}\right)
$$

of the w-vertex induced subgraphs $S[W]$ are $0.999 \eta^{2} \alpha(n / 2)$-absorber-resilient.

Our proof strategy for finding many perfect matchings will roughly go as follows. Suppose that $\boldsymbol{S}$ is a typical $\operatorname{STS}(n)$. We show that (the edge-set of) $\boldsymbol{S}$ can be decomposed into $(\alpha, \beta)$-good subgraphs, for some carefully chosen $\alpha, \beta$. Then, in each of these subgraphs, we show that one can approximately decompose its edges into perfect matchings. Together, we obtain our desired collection of $(1-o(1)) n / 2$ perfect matchings.

One might wonder why we need this intermediate stage of partitioning $S$, instead of working with $\boldsymbol{S}$ directly. It is necessary because 'small bits' of $\boldsymbol{S}$ behave like the binomial random hypergraph model (recall Lemma 2.6), so we can borrow some tools and ideas from this well-studied model in order to construct our matchings.

Now we state our key lemmas, which together imply Theorem 1.2.

Lemma 3.5. There is $r \in \mathbb{N}$ such that the following holds. Suppose $n$ is congruent to 1 or 3 mod 6 , and let $\boldsymbol{S}$ be a uniformly random $\boldsymbol{S T S}(n)$. Then a.a.s. the edge-set of $\boldsymbol{S}$ can be partitioned into $r$ spanning subgraphs that are $(1 / r, o(1))$-good.

Lemma 3.6. For any $\alpha=\Omega(1)$ and $n \equiv 0 \bmod 3$, every $n$-vertex $(\alpha, o(1))$-good linear 3-graph $S$ (that is, a partial Steiner triple system) has $(\alpha / 2-o(1)) n$ disjoint perfect matchings.

The proof of Lemma 3.5 mainly comes down to studying the robust absorber-resilience property of goodness. (The almost regularity and pseudorandomness properties can be proved in a fairly straightforward fashion using Theorem 2.4 and Lemma 2.6). For the robust absorber-resilience property, we will use Theorem 2.4 and Lemma 2.6 in combination with a hypergraph generalisation of the sparse regularity lemma (to be stated in Section 4) and a hypergraph generalisation of the resolution of the KŁR conjecture by Conlon, Gowers, Samotij, and Schacht (to be stated in Section 4 as well). Roughly speaking, this allows us to reduce certain problems about subgraphs of a random hypergraph to problems about dense hypergraphs, and the latter case is much more tractable to study. One complication is that even though robust absorber-resilience is a property of subgraphs of a random hypergraph, an absorber is a rooted structure. We will need some additional tricks to reduce the situation to one where the KŁR conjecture can actually be applied. The details of the proof will be presented in Section 7 . 
Concerning Lemma 3.6, one may wonder how the robust absorber-resilience property of goodness could be strong enough to produce an approximate decomposition of $S$ into perfect matchings. Naïvely, it seems that after we have found only $0.001 \alpha n / 2$ perfect matchings, this property no longer gives us any guarantee for the existence of absorbers in the remaining edges. Indeed, the proof of Lemma 3.6 would be much simpler if the robust absorber-resilience property of goodness could be strengthened to guarantee that $S$ is $D$-absorber-resilient for $D=o(n)$. Unfortunately, it is not clear how to prove that such a strong property holds in a typical STS $(n)$, even with the KŁR conjecture in hand. Instead, we employ a random partitioning trick inspired by the work of Ferber, Kronenberg, and Long [11]. We partition the edges of $\boldsymbol{S}$ into many subgraphs with different roles, some of which are used to find almost-perfect matchings and some of which are used to complete almost-perfect matchings into perfect matchings (using absorbers). The latter subgraphs contain only a small fraction of the edges of $S$ but have comparatively high degree (this is possible because each of these subgraphs has a very small number of vertices). We then need only a weak absorber-resilience property for these smaller subgraphs. The details of the proof will be presented in Section 6.

\section{Sparse regularity and the KKR conjecture for hypergraphs}

Kohayakawa and Rödl [27] proved a sparse version of the so-called regularity lemma for graphs. In this paper, for the proofs of Lemmas 3.5 and 3.6, we will need a generalisation of the sparse regularity lemma to hypergraphs. Fortunately, while the general hypergraph regularity lemma is much more complicated to prove (and state) than the graph regularity lemma, for our purposes (embedding linear hypergraphs) we need only a sparse version of the 'weak' hypergraph regularity lemma (see, for example, [28, Theorem 9]). To state our sparse regularity lemma for hypergraphs, we need a few definitions.

Definition 4.1. Let $\varepsilon, \eta>0$ and $0 \leq p \leq 1$ be arbitrary parameters.

- Density: Consider disjoint vertex sets $X_{1}, \ldots, X_{r}$ in an $r$-graph $G$. Let $e\left(X_{1}, \ldots, X_{r}\right)$ be the number of edges with a vertex in each $X_{i}$, and let

$$
d\left(X_{1}, \ldots, X_{r}\right)=\frac{e\left(X_{1}, \ldots, X_{r}\right)}{\left|X_{1}\right| \ldots\left|X_{r}\right|}
$$

be the density between $X_{1}, \ldots, X_{r}$.

- Regular tuples: An r-graph $G$ is said to be $r$-partite if its vertex set $V(G)$ consists of a partition $V(H)=V_{1} \cup \ldots \cup V_{r}$ into $r$ parts in such a way that each of its edges intersects each $V_{i}$ in exactly one vertex. An $r$-partite $r$-graph with parts $V_{1}, \ldots, V_{r}$ is $(\varepsilon, p)$-regular if, for every $V_{1}^{\prime} \subseteq V_{1}, \ldots, V_{r}^{\prime} \subseteq V_{r}$ with $\left|V_{i}^{\prime}\right| \geq \varepsilon\left|V_{i}\right|$, the density $d\left(V_{1}^{\prime}, \ldots, V_{r}^{\prime}\right)$ of edges between $V_{1}^{\prime}, \ldots, V_{r}^{\prime}$ satisfies

$$
\left|d\left(V_{1}^{\prime}, \ldots, V_{r}^{\prime}\right)-d\left(V_{1}, \ldots, V_{r}\right)\right| \leq \varepsilon p .
$$

- Regular partitions: A partition of the vertex set of a $r$-graph into $t$ parts $V_{1}, \ldots, V_{t}$ is said to be $(\varepsilon, p)$-regular if it is an equipartition and for all but at most $\varepsilon t^{r} r$-tuples $\left(V_{i_{1}}, \ldots, V_{i_{r}}\right)$, the induced $r$-partite r-graph between $V_{i_{1}}, \ldots, V_{i_{r}}$ is $(\varepsilon, p)$-regular.

- Upper-uniformity: An r-graph $G$ is $(\eta, p, D)$-upper-uniform if for any choice of disjoint subsets $U_{1}, \ldots, U_{r}$ with $\left|U_{1}\right|, \ldots,\left|U_{r}\right| \geq \eta|V(G)|$, we have $d\left(U_{1}, \ldots, U_{2}\right) \leq D p$.

Note that upper-uniformity is a weaker property than upper-quasirandomness: if a 3-graph $G$ is $(o(1), p)$-upper-quasirandom, then it is $(o(1), p, 1+o(1))$-upper-uniform. Now, our hypergraph regularity lemma is as follows. We omit its proof since it is straightforward to adapt a proof of the sparse graph regularity lemma (see [27] for a sparse regularity lemma for graphs; and see [28, Theorem 9] for a weak regularity lemma for dense hypergraphs). 
Lemma 4.2. For every $\varepsilon, D>0$ and every positive integer $t_{0}$, there exist $\eta>0$ and $T \in \mathbb{N}$ such that for every $p \in[0,1]$, every $(\eta, p, D)$-upper-uniform $r$-graph $G$ with at least $t_{0}$ vertices admits an $(\varepsilon, p)$-regular partition $V_{1}, \ldots, V_{t}$ of its vertex set into $t_{0} \leq t \leq T$ parts.

We will use Lemma 4.2 for several different purposes. Crucial to all of these is the notion of a cluster hypergraph, which is a dense hypergraph that encodes the large-scale structure of a regular partition. From now on, we return to considering only 3-graphs.

Definition 4.3. Given an $(\varepsilon, p)$-regular partition $V_{1}, \ldots, V_{t}$ of the vertex set of a 3-graph $G$, the cluster hypergraph is the 3-graph whose vertices are the clusters $V_{1}, \ldots, V_{t}$, with an edge $\left\{V_{i}, V_{j}, V_{k}\right\}$ if $d\left(V_{i}, V_{j}, V_{k}\right)>2 \varepsilon p$ and the induced tripartite 3-graph between $V_{i}, V_{j}$ and $V_{k}$ is $(\varepsilon, p)$-regular.

If the regularity lemma is applied with small $\varepsilon$ and large $t_{0}$, the cluster hypergraph approximately inherits certain density properties from the original graph $G$, as follows.

Lemma 4.4. Fix sufficiently small $\varepsilon>0$ and sufficiently large $t_{0} \in \mathbb{N}$, and let $G$ be an $n$-vertex ( $p, o(1)$ )-upper-quasirandom 3-graph. Let $G^{\prime} \subseteq G$ be a spanning subgraph with minimum degree at least $\delta p\left(\begin{array}{l}n \\ 2\end{array}\right)$. Let $\mathcal{R}$ be the $t$-vertex cluster 3 -graph obtained by applying the sparse regularity lemma to $G^{\prime}$ with parameters $t_{0}, p$, and $\varepsilon$. Then all but $\sqrt{\varepsilon} t$ vertices of $\mathcal{R}$ have degrees at least $\left(\delta-3 \sqrt{\varepsilon}-3 / t_{0}\right)\left(\begin{array}{c}t \\ 2\end{array}\right)$.

The proof of Lemma 4.4 is by a standard counting argument. It is a special case of Lemma 4.8, to appear in the next subsection (so we defer the proof until then).

An immediate application of Lemma 4.4 is the fact that high-degree subgraphs of upper-quasirandom 3-graphs have a 'rich' vertex subset $Z$ such that most vertices outside $Z$ have reasonably high degree into $Z$. This will be important for the proof of Lemma 3.6.

Lemma 4.5. For any $\delta>0$ and any $\varepsilon>0$ that is sufficiently small relative to $\delta$, there is $\xi>0$ such that the following holds. Let $G$ be an ( $p, o(1))$-upper-quasirandom 3-graph. Let $G^{\prime} \subseteq G$ be a spanning subgraph with minimum degree $\delta p\left(\begin{array}{l}n \\ 2\end{array}\right)$. Then there is a $2 \varepsilon n$-vertex subset $U \subseteq V(G)$ such that the following conditions hold:

1. For all but at most $2 \sqrt{\varepsilon} n$ vertices $v$, there are at least $\xi\left(\begin{array}{c}n \\ 2\end{array}\right)$ p edges of $G^{\prime}$ containing $v$ and two vertices of $U$,

2. Every subset of $|U|-\xi n$ vertices of $U$ induces at least one edge of $G^{\prime}$.

Proof. Apply the sparse regularity lemma (Lemma 4.2) to $G^{\prime}$ with small $\varepsilon$ and large $t_{0}$, and let $U$ contain a $2 \varepsilon$-fraction of each cluster. Let $\mathcal{R}$ be the cluster 3-graph (with $t \leq T$ vertices).

By Lemma 4.4, if $\varepsilon$ is small enough and $t_{0}$ is large enough, all but $\sqrt{\varepsilon} t$ clusters $V_{i}$ have degree at least $(\delta / 2)\left(\begin{array}{l}t \\ 2\end{array}\right)$ in the cluster graph and, in particular, participate in an $(\varepsilon, p)$-regular triple $V_{i}, V_{j}, V_{q}$ with $d\left(V_{i}, V_{j}, V_{q}\right)>2 \varepsilon p$ (recall that we are assuming that $\varepsilon$ is small relative to $\delta$ ).

Now, take $\xi=\varepsilon\left(1 / T^{2}\right)$. To verify the first part of Lemma 4.5, we observe that for any cluster $V_{i}$ as above (participating in an $(\varepsilon, p)$-regular triple $V_{i}, V_{j}, V_{q}$ with $d\left(V_{i}, V_{j}, V_{q}\right)>2 \varepsilon p$ ), at least a $(1-\varepsilon)$ fraction of the vertices $v \in V_{i}$ satisfy the condition in the first part of Lemma 4.5. Indeed, let $V_{j}^{\prime}=V_{j} \cap U$ and $V_{q}^{\prime}=V_{q} \cap U$, and let $V_{i}^{\prime}$ be the set of vertices $v \in V_{i}$ for which there are fewer than $\varepsilon\left|V_{j}^{\prime}\right|\left|V_{j}^{\prime}\right| p$ edges containing $v$, a vertex in $V_{j}^{\prime}$, and a vertex in $V_{j}^{\prime}$. Then $d\left(V_{i}^{\prime}, V_{j}^{\prime}, V_{q}^{\prime}\right)<\varepsilon p$, and if we had $\left|V_{i}^{\prime}\right| \geq \varepsilon\left|V_{i}\right|$, this would contradict $(\varepsilon, p)$-regularity. So, $\left|V_{i}^{\prime}\right|<\varepsilon\left|V_{i}\right|$. Then, observe that $\varepsilon\left|V_{j}^{\prime}\right|\left|V_{j}^{\prime}\right| p \geq \xi\left(\begin{array}{c}n \\ 2\end{array}\right) p$.

For the second property, if we delete fewer than $\xi n$ vertices from $U$, then we still have an $\varepsilon$-fraction of each cluster $V_{i}$ and therefore have at least one edge.

\subsection{Refining an existing partition}

In our proof of Lemma 3.5, we will apply the sparse regularity lemma to a 3-graph whose vertices are already partitioned into a few different parts with different roles. It will be important that the regular partition in Lemma 4.2 can be chosen to be consistent with this existing partition. 
Lemma 4.6. Suppose that a 3-graph $G$ has its vertices partitioned into sets $U_{1}, \ldots, U_{h}$. In the $(\varepsilon, p)$ regular partition guaranteed by Lemma 4.2, we can assume that all but at most $\varepsilon$ ht of the clusters $V_{i}$ are contained in some $U_{j}$.

For the reader who is familiar with the proof of the regularity lemma, the proof of Lemma 4.6 is straightforward. Indeed, in order to prove the regularity lemma, one starts with an arbitrary partition and keeps refining it in a clever way. Nevertheless, for completeness, we include a short reduction from Lemma 4.2.

Proof of Lemma 4.6. Apply Lemma 4.2 with regularity parameter $\varepsilon^{2} / 2$. For each cluster $V_{i}$, order the vertices in $V_{i}$ according to the partition $U_{1}, \ldots, U_{h}$ (first the vertices from $U_{1}$, then from $U_{2}$, etc). Now, equipartition $V_{i}$ into $\lfloor 1 / \varepsilon\rfloor$ intervals $V_{i}^{1}, \ldots, V_{i}^{\lfloor 1 / \varepsilon\rfloor}$ with respect to this ordering. At most $h$ of these intervals intersect multiple $U_{j}$, and the $V_{i}^{q}$ form an $(\varepsilon, p)$-regular partition. (Strictly speaking some of the clusters may now have sizes differing by 2 instead of 1 , but we can move some vertices between clusters to correct this without having any material effect on the regularity of the partition.)

We also need a more technical version of Lemma 4.4 translating the degrees between the $U_{i}$ into degrees in the cluster graph. First, we generalise the definition of a cluster graph.

Definition 4.7. Given a 3-graph $G$ with vertex partition $U_{1}, \ldots, U_{h}$ and an $(\varepsilon, p)$-regular partition $V_{1}, \ldots, V_{t}$ of its vertices, the partitioned cluster graph $\mathcal{R}$ with threshold $\tau$ is the 3 -graph defined as follows. The vertices of $\mathcal{R}$ are the clusters $V_{i}$ that are completely contained in some $U_{j}$, with an edge $\left\{V_{i}, V_{j}, V_{k}\right\}$ if $d\left(V_{i}, V_{j}, V_{k}\right)>\tau p$ and the induced tripartite 3-graph between $V_{i}, V_{j}$ and $V_{k}$ is $(\varepsilon, p)$-regular.

Lemma 4.8. Fix sufficiently small $\varepsilon>0$ and sufficiently large $t_{0} \in \mathbb{N}$, and let $G$ be an $n$-vertex $(p, o$ (1))upper-quasirandom 3-graph with a partition $U_{1}, \ldots, U_{h}$ of its vertices into parts of sizes $u_{1}, \ldots, u_{h}$, respectively. Let $G^{\prime} \subseteq G$ be a spanning subgraph such that each $v \in U_{i}$ has $\operatorname{deg}_{U_{j}}(v) \geq \delta_{i j} p\left(\begin{array}{c}u_{j} \\ 2\end{array}\right)$ (where the degree here is with respect to $G^{\prime}$ ).

Let $\mathcal{R}$ be the partitioned cluster 3-graph with threshold $\tau$ obtained by applying Lemmas 4.2 and 4.6 to $G^{\prime}$ with parameters $t_{0}, p$ and $\varepsilon$. Let $t_{i}$ be the number of clusters contained in $U_{i}$, and let $\mathcal{U}_{i}$ be the set of clusters contained in $U_{i}$. Then each $t_{i} \geq\left(u_{i} / n\right) t-\varepsilon h t$, and for each $i, j$, all but $\sqrt{\varepsilon} t$ clusters $X \in \mathcal{U}_{i}$ have $\operatorname{deg}_{\mathcal{U}_{j}}(X) \geq \delta_{i j}\left(\begin{array}{c}t_{i} \\ 2\end{array}\right)-t^{2}\left(\tau+2 \varepsilon h+\sqrt{\varepsilon}+2 / t_{0}\right)$ in the cluster graph $\mathcal{R}$.

Note that Lemma 4.4 is actually a special case of Lemma 4.8 (taking the trivial partition and threshold $2 \varepsilon)$.

Proof of Lemma 4.8. The clusters in $\mathcal{U}_{i}$ comprise at most $t_{i}(n / t)$ vertices, so $\left|U_{i}\right|=u_{i} \leq t_{i}(n / t)+$ $\varepsilon h t(n / t)$. It follows that $t_{i} \geq\left(u_{i} / n\right) t-\varepsilon h t$ as claimed.

Let $\mathcal{W}$ be the set of all clusters $W$ in the $(\varepsilon, p)$-regular partition for which there are more than $\sqrt{\varepsilon}\left(\begin{array}{l}t \\ 2\end{array}\right)$ non- $(\varepsilon, p)$-regular triples $\left(V_{i}, V_{j}, W\right)$ containing $W$. There can be at most $3 \varepsilon\left(\begin{array}{l}t \\ 3\end{array}\right) /\left(\sqrt{\varepsilon}\left(\begin{array}{l}t \\ 2\end{array}\right)\right) \leq \sqrt{\varepsilon} t$ clusters in $\mathcal{W}$. Let $Z$ be the set of at most $\varepsilon h n$ vertices whose cluster does not appear in the cluster 3-graph (because the cluster was not completely contained in any $U_{i}$ ).

If a cluster $X \in \mathcal{U}_{i} \backslash \mathcal{W}$ has $\operatorname{deg}_{\mathcal{U}_{j}}(X)=d$ in the cluster 3-graph, then by $(p, o(1))$-upperquasirandomness, the number of edges of $G^{\prime}$ with a vertex in $X$ and two vertices in $U_{j}$ is at most

$$
\begin{aligned}
d(1+ & o(1)) p\left(\frac{n}{t}\right)^{3}+\sqrt{\varepsilon}\left(\begin{array}{l}
t \\
2
\end{array}\right) p\left(\frac{n}{t}\right)^{3}+\tau\left(\begin{array}{l}
t_{j} \\
2
\end{array}\right) p\left(\frac{n}{t}\right)^{3}+e_{G}\left(X, Z \cup X, U_{j}\right)+\sum_{W \in V(\mathcal{R})} e_{G}(X, W, W) \\
& \leq(1+o(1)) p\left(\frac{n}{t}\right)^{3}\left(d+t^{2}(\tau+\sqrt{\varepsilon})+t(\varepsilon h t+1)+t\right) .
\end{aligned}
$$

But by the degree assumption in $G^{\prime}$ and the fact that $u_{j} \geq t_{j}(n / t)$, this number is at least

$$
(n / t) \delta_{i j} p\left(\begin{array}{c}
u_{j} \\
2
\end{array}\right) \geq p \delta_{i j}\left(\begin{array}{c}
t_{j} \\
2
\end{array}\right)\left(\frac{n}{t}\right)^{3} .
$$


It follows that

$$
d \geq \delta_{i j}\left(\begin{array}{c}
t_{j} \\
2
\end{array}\right)-t^{2}(\tau+2 \varepsilon h+\sqrt{\varepsilon}+2 / t)
$$

\subsection{The KER conjecture}

One of the most powerful aspects of the sparse regularity method is that, for a subgraph $G$ of a typical outcome of a random graph, if we find a substructure in the cluster graph (which is usually dense and therefore comparatively easy to analyse), then a corresponding structure must also exist in the original graph $G$. For graphs, this was conjectured to be true by Kohayakawa, Łuczak, and Rödl [26] and was proved by Conlon, Gowers, Samotij, and Schacht [8]. We will need a generalisation to hypergraphs, which we again state in a more general form than we need, in case it is useful for future applications. First we need some definitions.

Definition 4.9. Consider an r-graph $H$ with vertex set $\{1, \ldots, k\}$, and let $\mathcal{G}(H, n, m, p, \varepsilon)$ be the collection of all $r$-graphs $G$ obtained in the following way. The vertex set of $G$ is a disjoint union $V_{1} \cup \cdots \cup V_{k}$ of sets of size $n$. For each edge $\left\{i_{1}, \ldots, i_{r}\right\} \in E(H)$, we add to $G$ an $(\varepsilon, p)$-regular $r$-graph with $m$ edges between $V_{i_{1}}, \ldots, V_{i_{r}}$. These are the only edges of $G$.

Definition 4.10. For $G \in \mathcal{G}(H, n, m, p, \varepsilon)$, let $\#_{H}(G)$ be the number of 'canonical copies' of $H$ in $G$, meaning the copy of the vertex $i$ must come from $V_{i}$.

Definition 4.11. The $r$-density $m_{r}(H)$ of an $r$-graph $H$ is defined as

$$
m_{r}(H)=\max \left\{\frac{e\left(H^{\prime}\right)-1}{v\left(H^{\prime}\right)-r}: H^{\prime} \subseteq H \text { with } v\left(H^{\prime}\right)>r\right\} .
$$

Now, the hypergraph version of the KŁR conjecture is as follows.

Theorem 4.12. For every linear $r$-graph $H$ (that is, an $r$-graph where every two edges intersect on at most one vertex) and every $d>0$, there exist $\varepsilon, \xi>0$ with the following property. For every $\eta>0$, there is $C>0$ such that if $p \geq C N^{-1 / m_{r}(H)}$, then with probability $1-e^{-\Omega\left(N^{r} p\right)}$, the following holds in $\boldsymbol{G} \in \mathrm{H}^{r}(N, p)$. For every $n \geq \eta N, m \geq d p n^{r}$ and every subgraph $G^{\prime}$ of $\boldsymbol{G}$ in $\mathcal{G}(H, n, m, p, \varepsilon)$, we have

$$
\#_{H}\left(G^{\prime}\right)>\xi\left(\frac{m}{n^{r}}\right)^{e(H)} n^{v(H)} .
$$

The proof of Theorem 4.12 is almost exactly the same as the proof of [8, Theorem 1.6(i)]. In Section Appendix A, we will describe the exact changes one needs to make in order to turn the proof in [8] into a proof of Theorem 4.12.

\section{Almost-perfect matchings}

For the proof of Lemma 3.6, we will need multiple different ways to find almost-perfect matchings, which we will then be able to complete into perfect matchings using absorbers. The first result we will need is that high-degree subgraphs of upper-quasirandom 3-graphs have almost-perfect matchings.

Lemma 5.1. Let $G$ be an ( $p, o(1))$-upper-quasirandom 3-graph. Let $G^{\prime} \subseteq G$ be a spanning subgraph with minimum degree at least $0.9 p\left(\begin{array}{l}n \\ 2\end{array}\right)$. Then $G^{\prime}$ has a matching covering all but o $(n)$ vertices.

Proof. For sufficiently large $n^{\prime}$, every $n^{\prime}$-vertex 3-graph with minimum degree at least $0.8\left(\begin{array}{c}n^{\prime} \\ 2\end{array}\right)$ has a perfect matching; see for example [14]. So by Lemma 4.4, if we apply the sparse regularity lemma (Lemma 4.2) to $G^{\prime}$ with small $\varepsilon$ and large $t_{0}$, we can find a matching covering $t-2 \sqrt{\varepsilon} t$ vertices of the cluster graph. In each corresponding triple of clusters $V_{i}, V_{j}, V_{q}$, we can greedily find a matching with 
$(1-\varepsilon)(n / t)$ vertices, and we can combine these to get a matching in $G^{\prime}$ covering $n-3 \sqrt{\varepsilon} n$ vertices. Since $\varepsilon$ was arbitrary, this implies that we can find a matching covering all but $o(n)$ vertices.

The second result we need is that almost-regular 3-graphs can be almost-partitioned into almostperfect matchings, and moreover the leftover vertices in each matching can be assumed to be 'welldistributed'.

Theorem 5.2. Fix $\alpha \in[0,1]$, and consider a linear 3-graph $S$ with all degrees $\alpha n \pm o(n)$. Then $S$ has $\alpha n-o(n)$ edge-disjoint matchings $M_{1}, \ldots, M_{\alpha n-o(n)}$, each with $n / 3-o(n)$ edges, such that every vertex of $S$ appears in all but $o(n)$ of the $M_{i}$.

Theorem 5.2 is a simple consequence of the following theorem of Pippenger and Spencer [37], proved using a Rödl-nibble-type argument, which we reproduce below.

Theorem 5.3. Fix $\alpha \in[0,1]$, and consider a linear 3-graph $S$ with all degrees $\alpha n \pm o(n)$. Then the edges of $S$ can be partitioned into $\alpha n+o(n)$ edge-disjoint matchings.

Proof of Theorem 5.2. Fix any $\varepsilon>0$, which we treat as constant. Consider a partition of edge-disjoint matchings as guaranteed by Theorem 5.3. Note that each vertex appears in $\alpha n+o(n)$ of the matchings, by the almost-regularity condition on $S$. Let $Q$ be the number of matchings with fewer than $n / 3-\varepsilon n$ edges, so that the total number of edges covered by all the matchings is $e(S) \leq Q(n / 3-\varepsilon n)+(\alpha n+o(1)-Q)(n / 3)=$ $\alpha n^{2} / 3-Q \varepsilon n+o\left(n^{2}\right)$. But by the degree condition, we have $e(S)=\alpha n^{2} / 3+o\left(n^{2}\right)$, so $Q=o(n)$.

Now, deleting the $Q$ matchings with fewer than $n / 3-\varepsilon n$ edges, we obtain a collection of $\alpha n+o(n)-Q=$ $\alpha n-o(n)$ edge-disjoint matchings, each with at least $n / 3-\varepsilon n$ edges, such that each vertex appears in $\alpha n+o(n)-Q$ of the matchings, which is all but $o(n)$ of them. Since $\varepsilon$ could have been taken arbitrarily small, the desired result follows.

\section{Packing in good systems}

In this section, we prove Lemma 3.6. First, we show how to partition our 3-graph into subgraphs with certain 'nice' properties.

\subsection{Partitioning for packing}

The majority of the edges of our 3-graph $S$ will go into subgraphs $G_{1}, \ldots, G_{\ell}$. These subgraphs will have vertex sets $U_{1}, \ldots, U_{\ell}$ that each comprise almost all the vertices of $S$, but they will be rather sparse (each containing approximately a $1 / \ell$ fraction of the edges of $S$ ). Eventually, we will use Theorem 5.2 to find an almost-perfect packing of almost-perfect matchings in each of these subgraphs.

Some of the remaining edges will go into subgraphs $F_{1}, \ldots, F_{\ell}$, where the vertex set $W_{i}$ of each $F_{i}$ is complementary to the vertex set of $G_{i}$. Despite each $F_{i}$ having fewer edges than $G_{i}$, the degrees in $F_{i}$ will be much higher than the degrees in $G_{i}$ (this will be possible because the $W_{i}$ will be quite small, and $F_{i}$ will contain almost all the edges of $S$ within $W_{i}$ ).

Many of the edges that still remain will go into subgraphs $H_{1}, \ldots, H_{\ell}$, whose purpose is to serve as a 'bridge' between $G_{i}$ and $F_{i}$. For each $i \leq \ell$, after finding our almost-perfect matchings in $G_{i}$, we will use $H_{i}$ to extend each matching to cover all of $G_{i}$ and some of $F_{i}$, after which we can iteratively complete all of our matchings using absorber-resilience properties of $F_{i}$. The details of the properties we will need are summarised in the following lemma. Say that a graph is $(R, D)$-absorber-resilient if it is $D$-absorber-resilient after deleting any choice of at most $R$ vertices.

Lemma 6.1. For any fixed $\alpha$, consider an $(\alpha, o(1))$-good linear 3-graph $S$ with $n$ vertices. Fix any ( sufficiently small) $\delta>0$, and let $\ell=\delta^{-5 / 2}$. Then there exists a constant $\kappa=\kappa(\alpha, \delta)>0$ and a partition of the edges of $S$ into $3 \ell+1$ subgraphs $G_{1}, \ldots, G_{\ell}, H_{1}, \ldots, H_{\ell}, F_{1}, \ldots, F_{\ell}$, and $Q$ (not necessarily induced or spanning) such that the following properties hold: 
1. Most of the edges are covered: At least a $(1-3 \sqrt{\delta})$-fraction of the edges of $S$ are in some $G_{i}$.

2. Controlling the sizes of $U_{i}$ and $W_{i}$ : For each $i$, the vertex sets $U_{i}=V\left(G_{i}\right)$ and $W_{i}=V\left(F_{i}\right)$ partition $V(S)$, and $\left|W_{i}\right|=\delta n+o(n)$.

3. The 3-graphs $G_{i}$ are almost regular: Each $G_{i}$ has all degrees in the range

$$
\left(\alpha(1-\delta)^{2}(1-2 \sqrt{\delta}) / \ell\right) n / 2 \pm o(n) .
$$

4. The 3-graphs $F_{i}$ are relatively dense: Each $F_{i}$ has all degrees at least $0.9999\left(\alpha \delta^{2}\right) n / 2$.

5. Many 'bridging' edges: For every vertex of $G_{i}$, there are $\Omega(n)$ edges of $H_{i}$ with one vertex in $U_{i}$ and two vertices in $W_{i}$.

6. The 3-graphs $F_{i}$ are absorber-resilient: Each $F_{i}$ is $\left(\kappa n, 0.9995\left(\alpha \delta^{2}\right)(n / 2)\right)$-absorber-resilient.

Before we prove Lemma 6.1, we briefly state and prove a lemma regarding absorber-resilience.

Lemma 6.2. For any fixed $\alpha, \delta>0$, consider an ( $\alpha, o(1))$-good linear 3-graph $S$ with $n$ vertices. There is $\kappa=\kappa(\alpha, \delta)>0$ such that if $U$ is a random subset of vertices of $S$, obtained by including each vertex with probability $\delta$ independently, then $S[U]$ is $\left(\kappa n, 0.9995\left(\alpha \delta^{2}\right)(n / 2)\right)$-absorber-resilient with probability at least $1-e^{-\Omega(n)}$.

Proof. Choose $\kappa$ sufficiently small such that $\left(\begin{array}{c}n \\ \kappa n\end{array}\right) \exp \left(-10^{-8}(\delta / 2)^{4} \alpha^{2} n\right)=e^{-\Omega(n)}$. By the Chernoff bound, we have $|U| \geq \delta n / 2$ with probability at least $1-e^{-\Omega(n)}$. Condition on such an outcome for $|U|$, so that $U$ is now a uniformly random set of vertices of this size. Now, the desired result follows from the robust-absorber resilience property of goodness, taking the union bound over all ways to delete up to $k n$ vertices from $U$.

Now we can prove Lemma 6.1.

Proof of Lemma 6.1. We will describe a random procedure to build the $G_{i}, H_{i}, F_{i}$ and show that the desired properties are satisfied with positive probability. It suffices to show that each of the six properties holds with probability strictly larger than (say) 5/6 individually; the result will then follow by a simple union bound.

For each $i \leq \ell$ and each $v \in V(S)$, put $v \in W_{i}$ with probability $\delta$ (independently for each $i, v$ ). Then, let $U_{i}=V(S) \backslash W_{i}$. By a simple application of the Chernoff bounds, we see that a.a.s. property 2 holds. Moreover, by Lemma 6.2, we obtain that a.a.s. each $S\left[W_{i}\right]$ is $\left(\kappa n, 0.9995\left(\alpha \delta^{2}\right)(n / 2)\right)$-absorberresilient. Since we will choose $F_{i}$ to be a spanning subgraph of $S\left[W_{i}\right]$, we will obtain that a.a.s. property 6 holds.

Now, we build the $G_{i}, H_{i}, F_{i}$. Do the following for each edge $e$, independently.

1. If $e$ is a subset of some $W_{i}$ (which happens with probability $p_{F}:=1-\left(1-\delta^{3}\right)^{\ell} \approx \delta^{3} \ell=\sqrt{\delta}$ ), then do the following:

(a) If $e$ is a subset of a unique $W_{i}$, put $e \in F_{i}$.

(b) Otherwise, put $e \in Q$.

2. Choose a uniformly random $i \leq \ell$, and choose $p_{H}$ to satisfy $p_{F}+p_{H}=2 \sqrt{\delta}$. If $e$ is not a subset of any $W_{j}$, then with probability $p_{H} /\left(1-p_{F}\right)$, do the following:

(a) If $e$ has one vertex in $U_{i}$ and two vertices in $W_{i}$, put $e$ in $H_{i}$.

(b) Otherwise, put $e \in Q$.

3. The probability we have not taken any of the previous actions is $p_{G}:=1-2 \sqrt{\delta}$. In this case, do the following (still with $i \leq \ell$ as a uniformly random index):

(a) If $e \subseteq U_{i}$, put $e$ in $G_{i}$.

(b) Otherwise, put $e$ in $Q$.

By the Chernoff bound, a.a.s. property 1 holds. 
Now, for a vertex $v$, let $d_{G_{i}}(v)$ be the number of edges $e \in S$ containing $v$, such that $e \backslash\{v\} \subseteq U_{i}$ and such that in the above procedure, the random index chosen for $e$ is $i$. So, if $v \in U_{i}$, then $d_{G_{i}}=\operatorname{deg}_{G_{i}}(v)$. Since $S$ is a partial Steiner triple system, the edges containing $v$ do not intersect other than in $v$, so $d_{G_{i}}(v)$ has a binomial distribution $\operatorname{Bin}\left(\alpha n / 2 \pm o(n),(1-\delta)^{2} p_{G} / \ell\right)$. By the Chernoff bound and the union bound, it follows that a.a.s. property 3 holds.

Next, if for some $i \leq \ell$ and edge $e \in E(S)$ we condition on the event that $e \subseteq W_{i}$, then the probability that $e$ is contained in some other $W_{j}$ is $p^{*}:=1-\left(1-\delta^{3}\right)^{\ell-1} \approx \sqrt{\delta}$. So, for every $i \leq \ell$ and vertex $v$, if we condition on the event that $v \in W_{i}$, then $\operatorname{deg}_{F_{i}}(v)$ has a binomial distribution $\operatorname{Bin}\left(\alpha n / 2 \pm o(1), \delta^{2}\left(1-p^{*}\right)\right)$; so by the Chernoff bound, a.a.s. property 4 holds (provided $\delta$ is sufficiently small).

Finally, for every $i \leq \ell$ and vertex $v$, if we condition on the event that $v \in U_{i}$, then $\operatorname{deg}_{F_{i}}(v)$ has a binomial distribution $\operatorname{Bin}(\Omega(n), \Omega(1))$; so by the Chernoff bound, a.a.s. property 5 holds.

\subsection{Absorbers}

Absorbers are the basic building blocks for a larger structure that will eventually allow us to complete an almost-perfect matching into a perfect matching. The relative positions of the absorbers in this structure will be determined by a 'template' with a 'resilient matching' property.

Lemma 6.3. For any sufficiently large $n$, there exists a 3-graph $T$ with 10 n vertices, maximum degree at most 40, and an identified set $Z$ of $2 n$ vertices such that if we remove any $n$ vertices from $Z$, the resulting hypergraph has a perfect matching. We call $T$ a resilient template, and we call $Z$ its flexible set.

Lemma 6.3 is an immediate consequence of [31, Lemmas 5.2 and 5.3] and is proved using a construction due to Montgomery [35]. Now, we will want to arrange absorbers in the positions prescribed by a resilient template, as follows.

Definition 6.4. An absorbing structure is a 3-graph $H$ of the following form. Consider a resilient template $T$, and put externally vertex-disjoint absorbers on each edge of $T$, introducing 18 new vertices for each. Then delete the edges of $T$. That is, the template just describes the relative positions of the absorbers; its edges are not actually in the absorbing structure.

Note that an absorbing structure with a flexible set of size $2 n$ has at most $10 n+18 \times 400 n / 3=O(n)$ vertices, at most $13 \times(400 n / 3)=O(n)$ edges, and maximum degree at most $40=O(1)$. An absorbing structure $H$ has the same crucial property as the resilient template $T$ that defines it: if we remove any half of the vertices of the flexible set $Z$, then what remains of $H$ has a perfect matching. Indeed, after this removal, we can find a perfect matching $M$ of $T$; then our perfect matching of $H$ can consist of the covering matching of the absorber on each edge of $M$ and the non-covering matching for the absorber on each other edge of $T$.

So, if we can find an absorbing structure $H$ with flexible set $Z$ in our 3-graph $S$, then to find a perfect matching, it suffices to find a matching that covers all the vertices outside $H$ and any half of the vertices in $Z$. For the proof of Lemma 3.6, we will be able to construct an absorbing structure with prescribed flexible set using absorber-resilience and the following simple lemma.

Lemma 6.5. Suppose an n-vertex 3-graph $G$ has the property that in every induced subgraph with $n-n^{\prime}$ vertices, there is an absorber rooted on every triple of vertices. Suppose also that $n^{\prime}$ is sufficiently large. Then given any subset $Z \subseteq V(G)$ of size $\omega(1)=|Z| \leq n^{\prime} /(18 \times 400 / 3)$, $G$ contains an absorbing structure with flexible set $Z$.

Proof. Let $q=|Z| / 2$, and fix a resilient template $T$ on an arbitrary set of $10 q$ vertices of $G$ (the edges of $T$ do not have to exist in $G$ ). Now, we can build our absorbing structure greedily, iteratively finding disjoint absorbers on each edge of $T$. Indeed, at any point, the non-rooted vertices of the absorbers found so far together comprise a total of at most $18 \times 400 q / 3 \leq n^{\prime}$ vertices. After deleting these vertices, we can still continue to find absorbers rooted on every desired triple of vertices. 
In the proof of Lemma 3.6, to find a perfect matching, we will choose $Z$ to be a 'rich' set of vertices as guaranteed by Lemma 4.5. Then we will be able to use Lemma 5.1 to find a matching covering almost all the vertices outside our absorbing structure, and the choice of $Z$ will allow us to extend this to a matching covering all the vertices outside the absorbing structure and half of $Z$. We will then use the absorbing structure to complete this into a perfect matching.

\subsection{Proof of Lemma 3.6}

Now, we combine the lemmas in the last two subsections to prove Lemma 3.6.

Proof of Lemma 3.6. Fix sufficiently small $\delta>0$ (which we will treat as constant for most of the proof). Consider subgraphs $G_{1}, \ldots, G_{\ell}, H_{1}, \ldots, H_{\ell}, F_{1}, \ldots, F_{\ell}$ and vertex sets $U_{1}, \ldots, U_{\ell}, W_{1}, \ldots, W_{\ell}$ as guaranteed by Lemma 6.1 .

Consider some $i \leq \ell$. Let $M_{1}, \ldots, M_{s}$ be a collection of $s=\left(\alpha(1-\delta)^{2}(1-2 \sqrt{\delta}) / \ell\right) n / 2-o(n)$ almost-perfect matchings in $G_{i}$, as guaranteed by Theorem 5.2. We will show that for each $j \leq s$, regardless of how $M_{1}, \ldots, M_{j-1}$ were previously completed, we can complete $M_{j}$ to a perfect matching using the edges in $H_{i}$ and $F_{i}$ that have not been used so far. We will be able to conclude that $S$ has $\alpha(1-\delta)^{2}(1-2 \sqrt{\delta}) n / 2-o(n)$ edge-disjoint perfect matchings, which implies the desired result (since $\delta$ could have been chosen to be arbitrarily small).

Now, fix an arbitrary ordering of the $o(n)$ vertices in $U_{i}$ that are not yet covered by $M_{j}$. For each such uncovered vertex $u$ in turn, choose an edge of $H_{i}$ that contains $u$ and two vertices of $W_{i}$, which has not already been used for a previous completion and which does not intersect any edges chosen for previous uncovered vertices. Add this edge to $M_{j}$. To see that it is possible to make this choice, note that there are $\Omega(n)$ edges in $H_{i}$ that contain $u$. Only $o(n)$ of these edges have been used to complete previous matchings, and only $o(n)$ of these edges intersect an edge that was previously chosen for a different uncovered vertex (the edges containing $u$ do not intersect in any vertices other than $u$, because $S$ is a partial Steiner triple system).

After doing this, $M_{j}$ covers all of $U_{i}$ and $o(n)$ vertices of $W_{i}$. Let $W_{i}^{\prime}$ be the set of unmatched vertices in $W_{i}$, and let $F_{i}^{\prime}$ be the subgraph consisting of all edges of $F_{i}\left[W_{i}^{\prime}\right]$ not used for previous matchings $M_{q}, q<j$. We now need to find a perfect matching in $F_{i}^{\prime}$.

First, note that if $\delta$ is sufficiently small, then $s$ (which is about $\alpha \delta^{5 / 2} n / 2$ ) is much less than the degrees in $F_{i}$ (which are about $\alpha \delta^{2} n / 2$ ). So, $F_{i}$ and $F_{i}^{\prime}$ have minimum degree at least $0.9998\left(\alpha \delta^{2}\right) n / 2$. Let $\kappa=\Omega(1)$ be as in the statement of Lemma 6.1, let $n^{\prime}=\min \left(\kappa n / 2,0.0003\left(\alpha \delta^{2}\right) n / 2\right)$, and note that by the absorber-resilience property of $F_{i}$ (property 6 in Lemma 6.1 ), $F_{i}^{\prime}$ has the property that in any induced subgraph obtained by deleting at most $n^{\prime}$ vertices, there is an absorber rooted on every triple of vertices.

By Lemma 4.5 (with sufficiently small $\varepsilon$ ) and the pseudorandomness property of goodness (which implies the necessary upper-quasirandomness condition), for some $\xi=\Omega$ (1), we can find a vertex set $Z^{\prime}$ with the following properties:

1. $\Omega(n) \leq\left|Z^{\prime}\right| \leq n^{\prime} /(2 \times 18 \times 400 / 3)$.

2. All but $0.01 \alpha \delta^{2} n$ vertices have degree $\Omega(n)$ into $Z^{\prime}$.

3. Every subset of $\left|Z^{\prime}\right|(1-\xi)$ vertices of $Z^{\prime}$ induces at least one edge.

Arbitrarily add vertices to obtain a set $Z \supseteq Z^{\prime}$ such that $|Z| / 2=\left|Z^{\prime}\right|(1-\xi)$. By the absorber-resilience properties of $F_{i}^{\prime}$ and Lemma 6.5, we can find an absorbing structure $H$ in $F_{i}^{\prime}$ with flexible set $Z$. Let $X=V(H) \backslash Z$.

Consider the (at most $0.01 \alpha \delta^{2} n$ ) bad vertices that do not have the guaranteed degree into $Z^{\prime}$. Since there are so few of these vertices, and the absorbing structure $H$ is so small compared to the degrees in $F_{i}^{\prime}$, we can greedily find a matching in $F_{i}^{\prime}$ avoiding $V(H)$ and covering all the bad vertices. Let $F_{i}^{\prime \prime}$ be obtained from $F_{i}^{\prime}$ by removing the matched vertices and all the vertices in $X \cup Z^{\prime}$. Then by Lemma 5.1, we can find a matching covering all but $o(n)$ vertices of $F_{i}^{\prime \prime}$. Let $Y$ be the set of uncovered vertices. 
Now, it suffices to find a perfect matching in $F_{i}^{\prime}\left[Y \cup X \cup Z^{\prime}\right]$. By the richness of $Z^{\prime}$, in $F_{i}^{\prime}$ we can greedily find a matching of $|Y|$ edges, each with a vertex in $Y$ and two vertices in $Z^{\prime}$. We can then greedily augment this matching with edges inside $Z^{\prime}$ until there are $\left|Z^{\prime}\right|(1-\xi)=|Z| / 2$ vertices of $Z^{\prime}$ uncovered. Finally, we can use the absorbing structure to finish the perfect matching.

\section{Goodness in random Steiner triple systems}

Let $\boldsymbol{S}$ be a uniformly random ordered $\operatorname{STS}(n)$, and let $N=\left(\begin{array}{l}n \\ 2\end{array}\right) / 3=(1+o(1)) n^{2} / 6$. We will show that $S_{\alpha N}$ is a.a.s. $(\alpha, o(1))$-good as long as $\alpha$ is a sufficiently small constant. This will suffice to prove Lemma 3.5, because we can partition $S$ into $r$ partial Steiner triple systems that each have the same distribution as $\boldsymbol{S}_{N / r}$ and then take a union bound.

\subsection{Almost-regularity}

The almost-regularity property of goodness follows immediately from Theorem 2.4 , taking $Q=\mathcal{O}_{\alpha N}^{\varepsilon, 1}$ and observing that every $S \in \mathcal{O}_{\alpha N}^{\varepsilon, 1}$ has the required property. (We could also give a direct proof by considering a random ordering of the edges of a Steiner triple system; see [31, Section 2.1].)

\subsection{Upper-quasirandomness}

In this subsection, we prove that $\boldsymbol{S}_{\alpha N}$ a.a.s. satisfies the quasirandomness property of goodness. Consider $q \in \mathbb{N}$ (which we will treat as a sufficiently large constant).

Let $G \sim \mathrm{H}^{3}(n, p)$ with $p=(\alpha / q)(1+10(\alpha / q)) / n$. By the Chernoff bound, with probability $1-$ $e^{-\Omega\left(n^{2}\right)}$, our random 3-graph $G$ has the property that $e_{G}(X, Y, Z) \leq(\alpha / q)|X||Y||Z| / n+11(\alpha / q)^{2} n^{2}$ for every triple of disjoint vertex subsets $X, Y, Z$. Denote this property by $\mathcal{P}$. By Lemma 2.6 (with $S=\emptyset$ ) and Theorem 2.4 (with no conditioning; that is, $\mathcal{Q}=\mathcal{O}_{\alpha N} \cup\{*\}$ ), $\mathcal{P}$ also holds a.a.s. in $\boldsymbol{S}_{\alpha N / q}$. By symmetry, in fact, it holds a.a.s. in $\boldsymbol{S}^{(\ell)}:=\boldsymbol{S}_{\alpha \ell N / q} \backslash \boldsymbol{S}_{\alpha(\ell-1) N / q}$ for each $\ell \leq q$.

So, a.a.s. for every triple of vertex subsets $X, Y, Z$ in $S_{\alpha N}$, we have

$$
e_{\boldsymbol{S}_{\alpha N}}(X, Y, Z)=\sum_{\ell \leq q} e_{\boldsymbol{S}^{(\ell)}}(X, Y, Z) \leq \alpha|X||Y||Z| / n+11\left(\alpha^{2} / q\right) n^{2} .
$$

Since $q$ could have been arbitrarily large, the desired result follows.

\subsection{Embedding absorbers}

To finish the proof of Lemma 3.5, we need to prove that the robust absorber-resilience property of goodness holds a.a.s. in $\boldsymbol{S}_{\alpha N}$. To do this, we use Theorem 2.4 in its full generality, conditioning on the almost-regularity of the first few steps of the triangle removal process.

Let $\alpha^{\prime}=\alpha / 2$, let $a$ be small enough for Theorem 2.4, and let

$$
\mathcal{Q}=\{*\} \cup\left\{S \in \mathcal{O}_{\alpha N}: S_{\alpha^{\prime} N} \in \mathcal{O}_{\alpha^{\prime} N}^{n^{-a}, 1}\right\} \supseteq \mathcal{O}_{\alpha N}^{n^{-a}, 1} .
$$

The plan is to use Lemma 2.6 and Theorem 4.12 to show that for any $S \in \mathcal{O}_{\alpha^{\prime} N}^{n^{-a}, 1}$, the triangle-removal process $\mathrm{R}\left(S, \alpha^{\prime} N\right)$ is extremely likely to produce a partial Steiner triple system that has certain properties that make it easy to find absorbers. We will then be able to use Theorem 2.4 to prove that $S_{\alpha N}$ is likely to have these properties as well. In combination with some much simpler facts about concentration of degrees in $S_{\alpha N}$, this will allow us to deduce the desired robust absorber-resilience property. Fix some $\beta>0$ that is very small compared to $\alpha$; and for every subset of vertices $W \subseteq[n]$, fix an equipartition $W=\pi_{A}(W) \cup \pi_{B}(W)$. Our main objective in this subsection is to prove the following claim. 
Claim 7.1. Consider any $S \in \mathcal{O}_{\alpha^{\prime} N}^{n^{-a}, 1}$, and let $\boldsymbol{G} \sim \mathrm{H}^{3}(n, p)$ with $p=\alpha^{\prime}\left(1+10 \alpha^{\prime}\right) / n$. If $\beta>0$ is sufficiently small, then for any subset $W$ of $w \geq \beta n$ vertices, the following holds with probability $1-e^{-\Omega\left(n^{2}\right)}$. Consider any spanning subgraphs $G^{\prime} \subseteq \boldsymbol{G}[W]$ and $S^{\prime} \subseteq S[W]$ such that

1. $S^{\prime}$ has minimum vertex degree at least $0.98\left(\alpha^{\prime} w^{2} / n^{2}\right)(n / 2)$, and

2. In $G^{\prime}$, for any vertex $v \in W$ and every $\beta^{3} n$-vertex subset $U$,

$$
\operatorname{deg}_{\pi_{A}(W) \backslash U}(v), \operatorname{deg}_{\pi_{B}(W) \backslash U}(v) \geq 0.98\left(\alpha^{\prime}\left(\frac{w / 2}{n}\right)^{2}\right) \frac{n}{2} .
$$

Then for any vertices $x, y, z \in W$, there is an absorber in $S^{\prime} \cup G^{\prime}$ rooted at $x, y, z$.

Before proving Claim 7.1 we show how it completes the proof of Lemma 3.5. We break down this deduction into some relatively simple claims. First, in $S_{\alpha N}$, the degrees into various subsets are typically quite well-behaved. Let $\gamma_{w}=0.0001(w / n)^{2} \alpha$.

Claim 7.2. A.a.s. $\boldsymbol{S}_{\alpha N}$ has the property that for any $w \geq \beta n$ and at least $\left(1-\exp \left(-10^{-8}(w / n)^{4}\right.\right.$ $\left.\left.\alpha^{2} n\right)\right)\left(\begin{array}{c}n \\ w\end{array}\right)$ of the w-vertex subsets $W$ :

1. All of the vertex degrees in $\boldsymbol{S}_{\alpha^{\prime} N}[W]$ are $\left(\alpha^{\prime} w^{2} / n^{2}\right)(n / 2) \pm \gamma_{w} n$, and

2. In $\left(\boldsymbol{S}_{\alpha N} \backslash \boldsymbol{S}_{\alpha^{\prime} N}\right)[W]$, every vertex $v$ has degree $\left(\alpha^{\prime}(w / 2)^{2} / n^{2}\right)(n / 2) \pm \gamma_{w} n$ into $\pi_{A}(W)$ and into $\pi_{B}(W)$; and there are $2\left(\alpha^{\prime}(w / 2)^{2} / n^{2}\right)(n / 2) \pm \gamma_{w} n$ edges containing $v$, a vertex in $\pi_{A}(W)$ and a vertex in $\pi_{B}(W)$.

Proof. Let $w \geq \beta n$, and consider a particular $w$-vertex set $W$. Observe that randomly reordering the edges and vertices of $\boldsymbol{S}$ does not change its distribution, so by a concentration inequality for the hypergeometric distribution (see for example [15, Theorem 2.10]) and the union bound, with probability at least $1-e^{-(3 / 2) \gamma_{w}^{2} n}$ the desired properties hold for $W$. By Markov's inequality, a.a.s. for every $w \geq \beta n$ the number of $W$ for which the properties fail to hold is at most $e^{-\gamma_{w}^{2} n}\left(\begin{array}{c}n \\ w\end{array}\right)=\exp \left(-10^{-8}(w / n)^{4} \alpha^{2} n\right)\left(\begin{array}{c}n \\ w\end{array}\right)$.

Next, the following claim summarises how to use Theorem 2.4 and Lemma 2.6 to turn Claim 7.1 into a fact about random Steiner triple systems.

Claim 7.3. A.a.s. $\boldsymbol{S}_{\alpha N}$ has the following property, provided $\beta$ is sufficiently small. Consider any $w \geq \beta n$ and any $w$-vertex subset $W$, and consider spanning subgraphs $S^{\prime \prime} \subseteq\left(\boldsymbol{S}_{\alpha N} \backslash \boldsymbol{S}_{\alpha^{\prime} N}\right)[W]$ and $S^{\prime} \subseteq S_{\alpha^{\prime} N}[W]$ such that

1. $S^{\prime}$ has minimum vertex degree at least $0.98\left(\alpha^{\prime} w^{2} / n^{2}\right)(n / 2)$, and

2. In $S^{\prime \prime}$, for any vertex $v \in W$ and every $\beta^{3} n$-vertex subset $U$, we have

$$
\operatorname{deg}_{\pi_{A}(W) \backslash U}(v), \operatorname{deg}_{\pi_{B}(W) \backslash U}(v) \geq 0.98\left(\alpha^{\prime}\left(\frac{w / 2}{n}\right)^{2}\right) \frac{n}{2} .
$$

Then for any vertices $x, y, z \in W$, there is an absorber in $S^{\prime} \cup S^{\prime \prime}$ rooted at $x, y, z$.

Proof. Note that the property in Claim 7.1 is a monotone decreasing property of $\boldsymbol{G}$ (depending on $S$ ). Then combine Lemmas 2.6 and Theorem 2.4 and Claim 7.1 (with $\mathcal{Q}$ as in the beginning of this subsection).

Now, given Claims 7.2 and 7.3, it is fairly immediate to deduce that $\boldsymbol{S}_{\alpha N}$ a.a.s. has the desired absorber-resilience property, as follows. Suppose that $\boldsymbol{S}_{\alpha N}$ satisfies the conclusions of Claims 7.2 and 7.3, and consider $w \geq \beta n$. Suppose for some $w$-vertex subset $W$ that $\boldsymbol{S}_{\alpha^{\prime} N}[W]$ and $\left(\boldsymbol{S}_{\alpha N} \backslash \boldsymbol{S}_{\alpha^{\prime} N}\right)[W]$ both satisfy the degree conditions in Claim 7.2 (this is true for at least $\left(1-\exp \left(-10^{-8}(w / n)^{4} \alpha^{2} n\right)\right)\left(\begin{array}{c}n \\ w\end{array}\right)$ of the choices of $\left.W\right)$. We want to show that $\boldsymbol{S}_{\alpha N}[W]$ is $D$-absorberresilient, for $D=0.999\left(\alpha w^{2} / n^{2}\right)(n / 2)$. Consider a subgraph $S \subseteq S_{\alpha N}[W]$ with minimum degree $D$. 
Observe that by the choice of $\gamma_{w}$, the hypergraph $S^{\prime}=S \cap S_{\alpha^{\prime} N}$ consisting of the edges of $S$ that appear in $\boldsymbol{S}_{\alpha^{\prime} N}$ has minimum degree at least

$$
0.999\left(\alpha \frac{w^{2}}{n^{2}}\right) \frac{n}{2}-4\left(\left(\alpha^{\prime}\left(\frac{w / 2}{n}\right)^{2}\right) \frac{n}{2} \pm \gamma_{w} n\right) \geq 0.98\left(\alpha^{\prime} \frac{w^{2}}{n^{2}}\right) \frac{n}{2}
$$

Next, let $S^{\prime \prime}=S \backslash S_{\alpha^{\prime} N}$, and consider any vertex $v$ and any set of $U$ of $\beta^{3} n$ other vertices of $W$. Since $S$ is a partial Steiner triple system, there are at most $\beta^{3} n$ edges of $S$ involving both $v$ and $U$; so for sufficiently small $\beta$, in $S^{\prime \prime}$ we have

$$
\begin{aligned}
\operatorname{deg}_{\pi_{A}(W) \backslash U}(v) & \geq 0.999\left(\alpha^{\prime} \frac{w^{2}}{n^{2}}\right) \frac{n}{2}-\left(\left(\alpha^{\prime} \frac{w^{2}}{n^{2}}\right)(n / 2) \pm \gamma_{w} n\right)-3\left(\left(\alpha^{\prime}\left(\frac{w / 2}{n}\right)^{2}\right) \frac{n}{2} \pm \gamma_{w} n\right)-\beta^{3} n \\
& \geq 0.98\left(\alpha^{\prime}\left(\frac{w / 2}{n}\right)^{2}\right) \frac{n}{2},
\end{aligned}
$$

and similarly $\operatorname{deg}_{\pi_{B}(W) \backslash U}(v) \geq 0.98\left(\alpha^{\prime}(w / 2)^{2} / n^{2}\right)(n / 2)$. So, in $S^{\prime}$ there is an absorber on every triple of vertices, concluding the proof of $D$-absorber-resilience.

It remains to prove Claim 7.1. As in the statement of Claim 7.1, consider any $S \in \mathcal{O}_{\alpha^{\prime} N}^{n^{-a}}$, , and let $\boldsymbol{G} \sim \mathrm{H}^{3}(n, p)$ with $p=\alpha^{\prime}\left(1+10 \alpha^{\prime}\right) / n$. The proof of Claim 7.1 will reduce to another claim about a family of auxiliary graphs obtained by contracting edges of subgraphs of $\boldsymbol{G}$. For a vertex set $W$ containing a vertex $v$, let $H_{S[W]}(v)$ be the set of all edges containing $v$ in $S[W]$.

Definition 7.4. Consider any subset $W$ of $w \geq \beta n$ vertices of $\boldsymbol{G}$, fix any $x, y, z \in W$, and consider any subsets $H_{x} \subseteq H_{S[W]}(x), H_{y} \subseteq H_{S[W]}(y), H_{z} \subseteq H_{S[W]}(z)$, each with $0.98\left(\alpha^{\prime} w^{2} / n^{2}\right)(n / 2)$ edges. Let $\boldsymbol{G}\left(W, x, y, z, H_{x}, H_{y}, H_{z}\right)$ be a 3-graph obtained from $\boldsymbol{G}[W \backslash\{x, y, z\}]$ by doing the following:

1. For $k=\beta^{4} n$, choose distinct vertices

$$
a_{1}^{x}, \ldots, a_{k}^{x}, b_{1}^{x}, \ldots, b_{k}^{x}, a_{1}^{y}, \ldots, a_{k}^{y}, b_{1}^{y}, \ldots, b_{k}^{y}, a_{1}^{z}, \ldots, a_{k}^{z}, b_{1}^{z}, \ldots, b_{k}^{z}
$$

such that for each $0 \leq i \leq k$, we have $\left\{x, a_{i}^{x}, b_{i}^{x}\right\} \in H_{x},\left\{y, a_{i}^{y}, b_{i}^{y}\right\} \in H_{y},\left\{z, a_{i}^{z}, b_{i}^{z}\right\} \in H_{z}$. If $\beta$ is sufficiently small, this can be done greedily, recalling that the edges of $H_{x}$ (respectively, of $H_{y}$ or of $H_{z}$ ) intersect only at $x$ (respectively, only at $y$ or at $z$ ).

2. Let $W_{A}$ (respectively, $W_{B}$ ) be the subset of $\pi_{A}(W)$ (respectively, $\pi_{B}(W)$ ) obtained by removing $x, y, z$ and all $3 k$ of the vertices chosen in the first step.

3. Then, for each $v \in\{x, y, z\}$, delete all edges containing a vertex of $H_{v}$, except those containing two vertices from $W_{A}$ and some $a_{i}^{v}$, or two vertices from $W_{B}$ and some $b_{i}^{v}$.

4. Finally, for each $v \in\{x, y, z\}$, contract each pair $\left\{a_{i}^{v}, b_{i}^{v}\right\}$ to a single vertex. Let $U_{v}$ be the set of newly contracted vertices.

Now, to prove Claim 7.1, it suffices to prove the following claim (observe that $2\left|U_{x}\right|+2\left|U_{y}\right|+2\left|U_{z}\right|+$ $3 \leq \beta^{3} n$ for sufficiently small $\beta$, and taking the union bound over all choices of $W, x, y, z, H_{x}, H_{y}, H_{z}$ costs us a factor of only $\left.e^{O(n)}\right)$.

Claim 7.5. For any $W, x, y, z, H_{x}, H_{y}, H_{z}$ as in Definition $7.4, \boldsymbol{G}^{\prime}:=\boldsymbol{G}\left(W, x, y, z, H_{x}, H_{y}, H_{z}\right)$ has the following property with probability $1-e^{-\Omega\left(n^{2}\right)}$. For any spanning subgraphs

$$
G_{A} \subseteq \boldsymbol{G}^{\prime}\left[W_{A} \cup U_{x} \cup U_{y} \cup U_{z}\right], \quad G_{B} \subseteq \boldsymbol{G}^{\prime}\left[W_{B} \cup U_{x} \cup U_{y} \cup U_{z}\right]
$$

with minimum vertex degree at least $0.98\left(\alpha(w / 2)^{2} / n^{2}\right)(n / 2)$, there are vertices $x^{\prime} \in U_{x}, y^{\prime} \in U_{y}$, $z^{\prime} \in U_{z}$ such that in both $G_{A}$ and $G_{B}$, there is a sub-absorber rooted on $x^{\prime}, y^{\prime}, z^{\prime}$. 
We will prove Claim 7.5 with the sparse regularity lemma (Lemma 4.2) and Theorem 4.12. Before doing this, we prove some final auxiliary lemmas. First, we need to know that absorbers are suitably sparse to apply Theorem 4.12. Recall the definition of 3-density from Definition 4.11.

Lemma 7.6. Consider an absorber and a contract, each rooted edge to a single vertex. Call the resulting 3-graph $H$ a contracted absorber. ( $H$ is equivalently obtained by gluing together two sub-absorbers on their rooted vertices.) Then $H$ has $m_{3}(H)<1$.

Proof. Note that $H$ has maximum degree 2. Let $H^{\prime}$ be a subgraph of $H$ with $q$ vertices of degree 2 . Then, $3 e\left(H^{\prime}\right) \leq v\left(H^{\prime}\right)+q$, and it follows that we can only have $\left(e\left(H^{\prime}\right)-1\right) /\left(v\left(H^{\prime}\right)-3\right) \geq 1$ if $q \geq 2 v\left(H^{\prime}\right)-6$. This is impossible if $v\left(H^{\prime}\right)>6$, so it suffices to consider subgraphs $H^{\prime}$ with up to 6 vertices. It then suffices to check by hand that every pair of edges spans at least 5 vertices, and every triple of edges spans at least 7 vertices.

Second, the sparse regularity lemma will give us a very dense cluster 3-graph, and we will need to be able to find absorbers in such a 3-graph.

Lemma 7.7. Let $F$ be an n-vertex 3-graph with degrees at least $0.96\left(\begin{array}{l}n \\ 2\end{array}\right)$, having an identified subset $U$ of at most $0.001 n$ vertices. If $n$ is sufficiently large, then for any $x, y, z, \in U$, there is a sub-absorber rooted at $x, y, z$ in which the only edges involving vertices of $U$ are the rooted ones.

Proof. Let $V=V(F)$, and consider any $x, y, z \in U$. Every vertex has degree at least $0.96\left(\begin{array}{c}n \\ 2\end{array}\right)-0.001 n^{2}$ into $V \backslash U$, so there are at least $\left(0.96\left(\begin{array}{c}n \\ 2\end{array}\right)-0.001 n^{2}\right)^{3}-o\left(n^{6}\right)$ choices of three disjoint edges, each containing one of $x, y$, and $z$ and two vertices in $V \backslash U$. For each such choice of three edges, there is a pair of edges in $U$ whose presence would yield a suitable sub-absorber, and a pair of edges in $F[V \backslash U]$ can contribute in this way to at most $(3 !)^{2}$ sub-absorbers. It follows that there are at least $\left(0.96\left(\begin{array}{c}n \\ 2\end{array}\right)-0.001 n^{2}\right)^{3} /(3 !)^{2}-$ $o\left(n^{6}\right)$ pairs of edges whose presence would yield a sub-absorber. But note that all but at most $\left(1-0.96^{2}\right)\left(\begin{array}{l}n \\ 3\end{array}\right)^{2}$ of the possible pairs of edges are present in $F$, and $(0.96 / 2 !-0.001)^{3} /(3 !)^{2}>$ $\left(1-0.96^{2}\right)(1 / 3 !)^{2}$, so for large $n$, there must be a sub-absorber as desired.

Now we can finally prove Claim 7.5, completing the proof of Lemma 3.5.

Proof of Claim 7.5. Consider all the triples of vertices intersecting $U_{x} \cup U_{y} \cup U_{z}$ except those with one vertex in $U_{x} \cup U_{y} \cup U_{z}$ and two vertices in $W_{A}$, or one vertex in $U_{x} \cup U_{y} \cup U_{z}$ and two vertices in $W_{B}$. None of these triples are edges in $\boldsymbol{G}^{\prime}$. Let $\boldsymbol{G}_{\text {extra }}$ be a random 3-graph, independent from $\boldsymbol{G}^{\prime}$, where each of these triples is included with probability $p:=\alpha^{\prime}\left(1+10 \alpha^{\prime}\right) / n$ independently. Now $\boldsymbol{G}^{\prime} \cup \boldsymbol{G}_{\text {extra }}$ is a standard binomial random 3-graph on the vertex set $U_{x} \cup U_{y} \cup U_{z} \cup W_{A} \cup W_{B}$ where each edge is present with probability $p$. The only purpose of the edges in $\boldsymbol{G}_{\text {extra }}$ is to put us in the setting for Theorem 4.12; we will not actually use these edges for anything.

Let $H$ be a contracted absorber, as defined in Lemma 7.6. By Theorem 4.12, there is $\varepsilon>0$ such that with probability $1-e^{-\Omega\left(n^{2}\right)}$ the random 3-graph $\boldsymbol{G}^{\prime} \cup \boldsymbol{G}_{\text {extra }}$ has the property that for any $n^{\prime}=\Omega(n)$ and any $m \geq 0.001 p\left(n^{\prime}\right)^{3}$, every subgraph $G^{\prime \prime} \subseteq \boldsymbol{G}^{\prime} \cup \boldsymbol{G}_{\text {extra }}$ in $\mathcal{G}\left(H, n^{\prime}, m, p, \varepsilon\right)$ has $\#_{H}\left(G^{\prime}\right)>0$. Also, by the Chernoff bound (basically as in Section 7.2), $\boldsymbol{G}$ is $(p, \beta)$-upper-quasirandom with probability $1-e^{-\Omega\left(n^{2}\right)}$.

Assuming that the above two properties hold, apply Lemmas 4.2 and 4.6 to $G_{A} \cup G_{B}$ with regularity parameter $\varepsilon>0$ that is small even compared to $\beta$, and with $t_{0} \geq 1 / \varepsilon$, to obtain a partitioned $t$-vertex cluster 3-graph $\mathcal{R}$ with threshold 0.001. Let $\mathcal{U}_{x}, \mathcal{U}_{y}, \mathcal{U}_{z}, \mathcal{W}_{A}, \mathcal{W}_{B}$ be the sets of clusters fully contained in $U_{x}, U_{y}, U_{z}, W_{A}, W_{B}$. By Lemma 4.8 , in both $\mathcal{R}_{A}:=\mathcal{R}\left[\mathcal{W}_{A} \cup \mathcal{U}_{x} \cup \mathcal{U}_{y} \cup \mathcal{U}_{z}\right]$ and $\mathcal{R}_{B}:=\mathcal{R}\left[\mathcal{W}_{B} \cup \mathcal{U}_{x} \cup \mathcal{U}_{y} \cup \mathcal{U}_{z}\right]$, all but $\sqrt{\varepsilon} t$ vertices have degree at least $0.97\left(\begin{array}{c}t / 2 \\ 2\end{array}\right)$. Delete at most $2 \sqrt{\varepsilon} t$ vertices to obtain induced subgraphs $\mathcal{R}_{A}^{\prime}, \mathcal{R}_{B}^{\prime}$ with minimum degree at least $0.96\left(\begin{array}{c}t / 2 \\ 2\end{array}\right)$.

Now, fix clusters $V_{x} \in \mathcal{U}_{x}, V_{y} \subseteq \mathcal{U}_{y}, V_{z} \subseteq \mathcal{U}_{z}$ that appear in both $\mathcal{R}_{A}^{\prime}$, $\mathcal{R}_{B}^{\prime}$, and apply Lemma 7.7 to $\mathcal{R}_{A}^{\prime}$ and to $\mathcal{R}_{B}^{\prime}$ to find sub-absorbers in the cluster graph rooted at $V_{x}, V_{y}, V_{z}$. These two sub-absorbers form a contracted absorber $\mathcal{A}$ in $\mathcal{R}$, so the edges between the clusters in $V(\mathcal{A})$ give us a subgraph of $\boldsymbol{G}^{\prime}$ 
in $\mathcal{G}\left(H, n^{\prime}, m, p, \varepsilon\right)$, for $n^{\prime}=n / t \geq n / T$ and $m \geq 0.001 p\left(n^{\prime}\right)^{3}$. It follows that we can find a canonical copy of a contracted absorber $H$, giving us a sub-absorber in $G_{A}$ and in $G_{B}$ rooted on the same three vertices.

\section{Concluding remarks}

There are many interesting further directions of research regarding random Steiner triple systems. Most obviously, Conjecture 1.1 is still open, though we imagine that an exact result would be quite difficult to prove. Perhaps a good starting point would be the methods of Kühn and Osthus [30], and Knox, Kühn and Osthus [25] for perfectly packing Hamilton cycles in random graphs and random tournaments.

A second interesting direction is to study the discrepancy of random Steiner triple systems. Combining the ideas in Section 7.2 and [31, Section 5.1.2], we have essentially proved the following theorem bounding the discrepancy of a random Steiner triple system, which may be of independent interest.

Theorem 8.1. Let $\boldsymbol{S}$ be a uniformly random order-n Steiner triple system. Then $\boldsymbol{S}$ a.a.s. satisfies the following property. For every triple of vertex subsets $X, Y, Z$, we have e $(X, Y, Z)=|X||Y||Z| / n+o\left(n^{2}\right)$.

We remark that an analogous theorem for Latin squares (with a stronger error term) was proved by Kwan and Sudakov [32]. See also the related conjectures in [33]. It would be very interesting if one could substantially improve the error term $o\left(n^{2}\right)$; we imagine the correct order of magnitude is $O(\sqrt{|X||Y||Z|})$, but a proof of this would require substantial new ideas.

Next, another interesting direction concerns containment and enumeration of subgraphs. Using Theorem 4.12 and the sparse regularity lemma (Lemma 4.2) in combination with Theorem 2.4 and Lemma 2.6, it is straightforward to prove the following result.

Theorem 8.2. Let $H$ be a 3-graph with $m_{3}(H)<1$. Then there is $\xi=\xi(H)>0$ such that a uniformly random $\operatorname{STS}(n)$ a.a.s. contains at least $\xi n^{v(H)-e(H)}$ copies of $H$.

We imagine that actually, the number of (labelled) copies of $H$ should a.a.s. be $(1 \pm o(1)) n^{v(H)-e(H)}$, but it is less obvious how to prove this. In particular, due to the 'infamous upper tail' issue (see [16]) and the fact that Theorem 2.4 only works with properties that hold with probability extremely close to 1 , any kind of upper bound on subgraph counts would require new ideas. We also remark that a lower bound was proved by Simkin [42] in the special case where $H$ is a Pasch configuration, using ideas from [31].

Another interesting question (also mentioned in [31]) is whether a random Steiner triple system typically contains a Steiner triple subsystem on fewer vertices. McKay and Wanless [34] proved that almost all Latin squares have many small Latin subsquares (see also [32]), but it was conjectured by Quackenbush [38] that most Steiner triple systems do not have proper subsystems. By comparison with a binomial random 3-graph, it seems likely that this conjecture is actually false, but it seems that substantial new ideas would be required to prove or disprove it.

Finally, one might try to generalise from Steiner triple systems to other classes of designs. It seems that the arguments in this paper should generalise in a straightforward fashion to Latin squares, proving that a random order- $n$ Latin square a.a.s. has $n-o(n)$ disjoint transversals (see [31] for a definition of Latin squares, a discussion of how the methods in Section 2 generalise to random Latin squares, and a discussion of the significance of transversals in Latin squares). It was actually conjectured by van Rees [43] that a random order- $n$ Latin square typically does not have a decomposition into $n$ disjoint transversals, although Wanless and Webb [44] observed that numerical observations seem more in line with the Latin squares analogue of Conjecture 1.1.

Also, a $(q, r, \lambda)$-design $(q>r)$ of order $n$ is a $q$-uniform hypergraph on the vertex set $[n]$ such that every $r$-set of vertices is included in exactly $\lambda$ edges. A $(q, r)$-Steiner system is a $(q, r, 1)$-design (so, a Steiner triple system is a $(3,2,1)$-design or, equivalently, a $(3,2)$-Steiner system, and a $d$-regular graph is a $(2,1, d)$-design). We expect that it should be fairly routine to adapt the definition of an absorber in the obvious way to prove that almost all $(q, r, \lambda)$-designs have a decomposition of almost all their 
edges into perfect matchings. As for Steiner triple systems, a design is said to be resolvable if it admits a decomposition into perfect matchings, and the general problem of whether resolvable block designs exist was only very recently solved by Keevash [21].

However, note that a 3-uniform perfect matching is actually a $(3,1)$-Steiner system, so as a sweeping generalisation of Conjecture 1.1, we might ask for which $r^{\prime} \leq r$ and $\lambda^{\prime} \leq \lambda$ do $(q, r, \lambda)$-designs typically admit a decomposition into spanning $\left(q, r^{\prime}, \lambda^{\prime}\right)$-designs of the same order. We note that in the case of regular graphs, a much stronger phenomenon occurs: there is a sense in which a random $\left(d_{1}+d_{2}\right)$ regular graph is 'asymptotically the same' as a random $d_{1}$-regular graph combined with a random $d_{2}$-regular graph (provided $d_{1}+d_{2}>2$; see [15, Section 9.5]).

\section{Appendix A. Adapting the proof of the KŁR conjecture to linear hypergraphs}

In their paper [8] on the KŁR conjecture for graphs, Conlon, Gowers, Samotij, and Schacht explicitly mentioned that their methods should extend to hypergraphs and that the generalisation should be particularly simple in the case of linear hypergraphs. Actually, the generalisation is so simple that we can describe in this short appendix exactly what changes to make to their proof of their Theorem 1.6 (i) (appearing in Section 2 of their paper) to turn it into a proof of Theorem 4.12. All theorem/lemma/section references are with respect to [8] unless noted otherwise.

- Change the notation ' $G(H)$ ' to ' $\#_{H}(G)$ ' (we changed this notation to avoid confusion with other notation in the paper).

- Change every instance of 'graph' to ' $r$-graph' and every instance of 'bipartite graph' to ' $r$-partite $r$-graph'.

- Change every instance of ' $m_{2}$ ' to ' $m_{r}$ '.

- Change every instance of ' $n^{2}$ ' to ' $n^{r}$ ', every instance of ' $n^{v(H)-2}$ ' to ' $n^{v(H)-r}$ ', and every instance of ' $N$, to ' $N$ ',

- The definition of $(\varepsilon, d)$-lower-regularity preceding the statement of Theorem 2.1 should be changed in the obvious way: an $r$-partite $r$-graph between sets $V_{1}, \ldots, V_{r}$ is $(\varepsilon, d)$-lower-regular if, for every $V_{1}^{\prime} \subseteq V_{1}, \ldots, V_{r}^{\prime} \subseteq V_{r}$ with $\left|V_{i}^{\prime}\right| \geq \varepsilon\left|V_{i}\right|$, the density $d\left(V_{1}^{\prime}, \ldots, V_{r}^{\prime}\right)$ of edges between $V_{1}^{\prime}, \ldots, V_{r}^{\prime}$ satisfies $d\left(V_{1}^{\prime}, \ldots, V_{r}^{\prime}\right) \geq d$.

- In the statement of Theorem 2.1, change 'let $H$ be an arbitrary graph' to 'let $H$ be an arbitrary linear $r$-graph'.

- At the beginning of the deduction of Theorem 1.6 (i) from Theorem 2.1, change the observation ' $m_{2}(H) \geq 1$ ' to ' $m_{r}(H) \geq 1 /(r-1)$ '.

- In the second displayed equation in the proof of Theorem 1.6 (i), change ' $e_{G_{N, p}}\left(W_{i}, W_{j}\right) \geq 2 p n^{2}$ for some $i j \in E(H)$ ' to ' $e_{G_{N, p}}\left(W_{i_{1}}, \ldots, W_{i_{r}}\right) \geq 2 p n^{r}$ for some $\left\{i_{1}, \ldots, i_{r}\right\} \in E(H)$ '.

- A one-sided counting lemma is presented without proof as Lemma 2.4. The corresponding generalisation for linear hypergraphs (with 'every graph $H$ ' replaced with 'every linear $r$-graph $H$ ') follows from essentially the same proof as [28, Lemma 10] (see also [9, Lemma 22]).

- In Section 2.2, change the definition of $C$ from ' $32 R L^{R} C^{\prime} /\left(\varepsilon^{2} d\right)$ ' to " $16 r R L^{R} C^{\prime} /\left(\varepsilon^{2} d\right)$ "; and in the proof of Claim 2.5, change the last display from ' $e(H) \cdot 2^{2 n} \exp \left(-\varepsilon^{2} d p_{s} n^{2} / 16\right)$ ' to ' $e(H) \cdot 2^{r n} \exp \left(-\varepsilon^{2} d p_{s} n^{r} / 16\right)$ '.

- In Section 2.2.2, the definition of $Z_{s}$ should be generalised in the obvious way:

$$
Z_{s}=\left\{e \in G\left(V_{i_{r}}, \ldots, V_{i_{r}}\right): \operatorname{deg}_{H^{\prime \prime}}\left(e, G, G_{s}^{\prime}\right) \geq \frac{\xi^{\prime}}{2} p_{s}^{e\left(H^{\prime \prime}\right)} n^{v(H)-r}\right\}
$$

where $\left\{i_{1}, \ldots, i_{r}\right\}$ is the edge of $H^{\prime}$ that is missing in $H^{\prime \prime}$, and $V_{i_{1}}, \ldots, V_{i_{r}}$ are the subsets of $V(G)$ corresponding to the vertices $i_{1}, \ldots, i_{r}$.

- In Case 1 of the proof of Claim 2.6, change 'there exist sets $X_{i} \subseteq V_{i}$ and $X_{j} \subseteq V_{j}$ with $\left|X_{i}\right|,\left|X_{j}\right| \geq \varepsilon n$ ' to 'there exist sets $X_{i_{1}} \subseteq V_{i_{1}}, \ldots X_{i_{r}} \subseteq V_{i_{r}}$ with $\left|X_{i_{1}}\right|, \ldots,\left|X_{i_{r}}\right| \geq \varepsilon n$ '. Also, change all instances of ' $\left(X_{i}, X_{j}\right)$ ' to ' $\left(X_{i_{1}}, \ldots, X_{i_{r}}\right)$ ', and change all instances of ' $\left|X_{i}\right|\left|X_{j}\right|$ to $\left|X_{i_{1}}\right| \ldots\left|X_{i_{r}}\right|$ '. 
- In Case 2 of the proof of Claim 2.6, change 'for every $i^{\prime}, j^{\prime} \in E(H)$ and every pair of sets $W_{i^{\prime}} \subseteq V_{i^{\prime}}$ and $W_{j^{\prime}} \subseteq V_{j^{\prime}} \in$ with $\left|W_{i^{\prime}}\right| \geq \varepsilon n$ and $\left|W_{j^{\prime}}\right| \geq \varepsilon n^{\prime}$ 'to 'for every $i_{1}^{\prime}, \ldots, i_{r}^{\prime} \in E(H)$ and any choice of $W_{i_{1}^{\prime}} \subseteq V_{i_{1}^{\prime}}, \ldots, W_{i_{r}^{\prime}} \subseteq V_{i_{r}^{\prime}}$ with $\left|W_{i_{1}^{\prime}}\right|, \ldots,\left|W_{i_{r}^{\prime}}\right| \geq \varepsilon n^{\prime}$ '. Also, change all instances of ' $\left(W_{i^{\prime}}, W_{j^{\prime}}\right)$ ' to ' $\left(W_{i_{1}^{\prime}}, \ldots, W_{i_{r}^{\prime}}\right)$ ', all instances of ' $\left|W_{i^{\prime}}\right|\left|W_{j^{\prime}}\right|$ ' to ' $\left|W_{i_{1}^{\prime}}\right| \ldots\left|W_{i_{r}^{\prime}}\right|$ ', and all instances of ' $\left(V_{i}, V_{j}\right)$ ' to ' $\left(V_{i_{1}}, \ldots, V_{i_{r}}\right)$ '.

Acknowledgements. Asaf Ferber's research was supported in part by NSF grants DMS-1954395 and DMS-1953799.

Matthew Kwan's research was supported in part by SNSF Project 178493 and NSF Award DMS-1953990.

We would like to thank the anonymous referees for their careful reading of the manuscript and their valuable comments.

Conflict of Interest: None.

\section{References}

[1] N. Alon, J.-H. Kim, and J. Spencer, 'Nearly perfect matchings in regular simple hypergraphs', Israel J. Math. 100 (1997), $171-187$.

[2] L. Babai, 'Almost all Steiner triple systems are asymmetric', Ann. Discrete Math. 7 (1980), 37-39, Topics on Steiner systems.

[3] S. Boucheron, G. Lugosi, and P. Massart, Concentration inequalities: A nonasymptotic theory of independence (Oxford University Press, 2013).

[4] D. Bryant and D. Horsley, 'Steiner triple systems without parallel classes', SIAM J. Discrete Math. 29(1) (2015), 693-696.

[5] P. J. Cameron, 'A generalisation of $t$-designs', Discrete Math. 309(14) (2009), 4835-4842.

[6] A. Cayley, 'On the triadic arrangements of seven and fifteen things', Phil. Mag. 37 (1850), 50-53.

[7] C. J. Colbourn and A. Rosa, 'Triple systems', Oxford University Press, 1999.

[8] D. Conlon, W. T. Gowers, W. Samotij, and M. Schacht, 'On the KŁR conjecture in random graphs', Israel J. Math. 203(1) (2014), 535-580.

[9] D. Conlon, H. Hàn, Y. Person, and M. Schacht, 'Weak quasi-randomness for uniform hypergraphs', Random Structures Algorithms. 40(1) (2012), 1-38.

[10] P. Erdôs and A. Rényi, 'On random graphs. I’, Publ. Math, Debrecen 6 (1959), 290-297.

[11] A. Ferber, G. Kronenberg, and E. Long, 'Packing, counting and covering Hamilton cycles in random directed graphs', Israel J. Math. 220(1) (2017), 57-87.

[12] D. A. Freedman, 'On tail probabilities for martingales', Ann. Probability 3 (1975), 100-118.

[13] A. Frieze and M. Krivelevich, 'Packing Hamilton cycles in random and pseudo-random hypergraphs', Random Structures \& Algorithms 41(1) (2012), 1-22.

[14] H. Hàn, Y. Person, and M. Schacht, 'On perfect matchings in uniform hypergraphs with large minimum vertex degree', SIAM J. Discrete Math. 23(2) (2009), 732-748.

[15] S. Janson, T. Łuczak, and A. Ruciński, Random graphs (Cambridge University Press, 2000).

[16] S. Janson and A. Ruciński, 'The infamous upper tail', Random Structures Algorithms 20(3) (2002), 317-342, Probabilistic methods in combinatorial optimization.

[17] A. Johansson, J. Kahn, and V. Vu, 'Factors in random graphs', Random Structures Algorithms 33(1) (2008), 1-28.

[18] R.M. Karp, 'Reducibility among combinatorial problems', Complexity of Computer Computations (1972), 85-103.

[19] P. Keevash, 'The existence of designs', arXiv preprint arXiv:1401.3665 (2014).

[20] P. Keevash, 'Counting designs', J. Eur. Math. Soc. (JEMS) 20(4) (2018), 903-927.

[21] P. Keevash, 'The existence of designs II', arXiv preprint arXiv:1802.05900 (2018).

[22] P. Keevash and R. Mycroft, 'A geometric theory for hypergraph matching', Mem. Amer. Math. Soc. 233(1098) (2015).

[23] T.P. Kirkman, 'On a problem in combinations', The Cambridge and Dublin Mathematical Journal (Macmillan, Barclay, and Macmillan), II: (1847) 191-204.

[24] T.P. Kirkman, 'QueryVI., lady's and gentleman's diary' (1850), 48.

[25] F. Knox, D. Kühn, and D. Osthus, 'Edge-disjoint Hamilton cycles in random graphs', Random Structures Algorithms 46(3) (2015), 397-445.

[26] Y. Kohayakawa, T. Łuczak, and V. Rödl, 'On $K^{4}$-free subgraphs of random graphs', Combinatorica 17(2) (1997), 173-213.

[27] Y. Kohayakawa and V. Rödl, Szemerédi's regularity lemma and quasi-randomness in Recent Advances in Algorithms and Combinatorics, CMS Books Math./Ouvrages Math. SMC, vol. 11 ( Springer, New York, 2003), 289-351.

[28] Y. Kohayakawa, B. Nagle, V. Rödl, and M. Schacht, 'Weak hypergraph regularity and linear hypergraphs', J. Combin. Theory Ser. B 100(2) (2010), 151-160.

[29] M. Krivelevich, B. Sudakov, V. H. Vu, and N. C. Wormald, 'Random regular graphs of high degree', Random Structures Algorithms 18(4) (2001), 346-363.

[30] D. Kühn and D. Osthus, 'Hamilton decompositions of regular expanders: a proof of Kelly's conjecture for large tournaments', Adv. Math. 237 (2013), 62-146. 
[31] M. Kwan, 'Almost all Steiner triple systems have perfect matchings', arXiv preprint arXiv:1611.02246 (2016).

[32] M. Kwan and B. Sudakov, 'Intercalates and discrepancy in random Latin squares', Random Structures Algorithms 52(2) (2018), 181-196.

[33] N. Linial and Z. Luria, 'Discrepancy of high-dimensional permutations', Discrete Anal. (2016), paper 11, 8.

[34] B. D. McKay and I. M. Wanless, 'Most Latin squares have many subsquares', J. Combin. Theory Ser. A 86(2) (1999), 322-347.

[35] R. Montgomery, 'Embedding bounded degree spanning trees in random graphs', arXiv preprint arXiv:1405.6559 (2014).

[36] P. Morris, 'Random Steiner triple systems', Master's thesis, Freie Universität Berlin (2017).

[37] N. Pippenger and J. Spencer, 'Asymptotic behavior of the chromatic index for hypergraphs', J. Combin. Theory Ser. A 51(1) (1989), 24-42.

[38] R. W. Quackenbush, 'Algebraic speculations about Steiner systems', Ann. Discrete Math. 7 (1980), 25-35, Topics on Steiner systems.

[39] D. K. Ray-Chaudhuri and R. M. Wilson, 'Solution of Kirkman's schoolgirl problem', in Combinatorics (Proc. Sympos. Pure Math.), vol. XIX (Univ. California, Los Angeles, Calif., 1968; Amer. Math. Soc., Providence, RI, 1971), 187-203.

[40] V. Rödl, 'On a packing and covering problem', European J. Combin. 6(1) (1985), 69-78.

[41] V. Rödl, A. Ruciński, and E. Szemerédi, 'An approximate Dirac-type theorem for k-uniform hypergraphs', Combinatorica 28(2) (2008), 229-260.

[42] M. Simkin, 'Methods for analyzing random designs', lecture notes: IIAS special day on combinatorial design theory, http://math.huji.ac.il/ michaels/files/papers/pasch_in_sts.pdf (2018).

[43] G. H. J. van Rees, 'Subsquares and transversals in Latin squares', Ars Combin. 29(B) (1990), 193-204, 12th British Combinatorial Conference (Norwich, 1989).

[44] I. M. Wanless and B. S. Webb, 'The existence of Latin squares without orthogonal mates', Des. Codes Cryptogr. 40(1) (2006), 131-135.

[45] L. Warnke, 'On the method of typical bounded differences', Combin. Probab. Comput. 25(2) (2016), 269-299. 